\title{
Novel experience induces persistent sleep-dependent plasticity in the cortex but not in the hippocampus
}

\author{
Sidarta Ribeiro ${ }^{1,2, *}$, Xinwu Shi ${ }^{3}$, Matthew Engelhard ${ }^{3}$, Yi Zhou ${ }^{3}$, \\ Hao Zhang ${ }^{3}$, Damien Gervasoni ${ }^{4}$, Shi-Chieh Lin ${ }^{3}$, Kazuhiro Wada ${ }^{3}$, \\ Nelson A. M. Lemos ${ }^{1,2}$ and Miguel A. L. Nicolelis ${ }^{1,3,5}$ \\ 1. Edmond and Lily Safra International Institute of Neuroscience of Natal (ELS-IINN), Natal, Brazil \\ 2. Department of Physiology, Universidade Federal do Rio Grande do Norte (UFRN), Natal, Brazil \\ 3. Department of Neurobiology, Duke University Medical Center, 101 Research Drive, Durham, USA \\ 4. CNRS UMR5167 - Université Claude Bernard Lyon 1, Faculté de Médecine Laënnec, Lyon, France \\ 5. Laboratory of Neural Ensemble Technology, École Polytechnique Fédérale de Lausanne, Lausanne, Switzerland \\ Review Editors: Robert Stickgold, Center for Sleep and Cognition, Harvard Medical School, USA \\ Sumimal Datta, Sleep and Cognitive Neuroscience Laboratory, Boston University School of Medicine
}

\begin{abstract}
Episodic and spatial memories engage the hippocampus during acquisition but migrate to the cerebral cortex over time. We have recently proposed that the interplay between slow-wave (SWS) and rapid eye movement (REM) sleep propagates recent synaptic changes from the hippocampus to the cortex. To test this theory, we jointly assessed extracellular neuronal activity, local field potentials (LFP), and expression levels of plasticity-related immediate-early genes (IEG) arc and zif-268 in rats exposed to novel spatio-tactile experience. Post-experience firing rate increases were strongest in SWS and lasted much longer in the cortex (hours) than in the hippocampus (minutes). During REM sleep, firing rates showed strong temporal dependence across brain areas: cortical activation during experience predicted hippocampal activity in the first post-experience hour, while hippocampal activation during experience predicted cortical activity in the third post-experience hour. Four hours after experience, IEG expression was specifically upregulated during REM sleep in the cortex, but not in the hippocampus. Arc gene expression in the cortex was proportional to LFP amplitude in the spindle-range $(10-14 \mathrm{~Hz})$ but not to firing rates, as expected from signals more related to dendritic input than to somatic output. The results indicate that hippocampo-cortical activation during waking is followed by multiple waves of cortical plasticity as full sleep cycles recur. The absence of equivalent changes in the hippocampus may explain its mnemonic disengagement over time.
\end{abstract}

\section{INTRODUCTION}

Memory consolidation requires two consecutive and distinct steps: neural reactivation for short-term recall and synaptic remodeling for long-term storage (Hebb, 1949). The consolidation of spatial and episodic memories is sleep-dependent (Peigneux et al., 2004; Stickgold et al., 2001), requires protein synthesis during sleep (Gutwein et al., 1980), and is characterized by both hippocampal disengagement and cortical engagement over time (Frankland and Bontempi, 2005; Izquierdo and Medina, 1997; Squire et al., 1993). Hippocampo-cortical reactivation during slow-wave sleep (SWS) (Pavlides and Winson, 1989; Peigneux et al., 2004; Ribeiro et al., 2004; Wilson and McNaughton, 1994) and upregulation during rapid eye movement (REM) sleep of calcium-dependent gene zif-268 (Ribeiro et al., 1999, 2002), a transcription factor (Wisden et al., 1990) with anterograde effects (Petersohn et al., 1995; Thiel et al., 1994) required for the consolidation of several kinds of memory (Bozon et al., 2003; Jones et al.,

* Correspondence: Edmond and Lily Safra International Institute of Neuroscience of Nata (ELS-IINN), Rua Professor Francisco Luciano de Oliveira 2460, Bairro Candelaria, Natal, RN, 59066-060, Brazil.e-mail: ribeiro@natalneuro.org.br

Received: 15 August 2007; paper pending published: 01 September 2007; accepted: 01 September 2007; published online: 15 October 2007.

Full citation: Frontiers in Neuroscience. (2007) vol. 1, iss. 1,43-55.

Copyright: () 2007 Ribeiro, Shi, Engelhard, Zhou, Zhang, Gervasoni, Lin, Wada, Lemos and Nicolelis. This is an open-access article subject to an exclusive license agreement between the authors and the Frontiers Research Foundation, which permits unrestricted use, distribution, and reproduction in any medium, provided the original authors and source are credited.
2001), suggest that the two major sleep states perform complementary mnemonic functions: neural reactivation during SWS and transcriptional storage during REM sleep (Ribeiro and Nicolelis, 2004; Ribeiro et al., 2004). In support of this view, it has recently been shown (Ulloor and Datta, 2005) that post-training REM sleep is associated with increased levels of the activity-regulated cytoskeleton-associated protein (Arc), a calcium-dependent immediate early gene (IEG) directly involved in synaptic remodeling (Guzowski et al., 2000; Lyford et al., 1995). Based on the fact that long-term potentiation induction in the hippocampus during waking (WK) leads to zif-268 gene upregulation in the cortex during subsequent REM sleep (Ribeiro et al., 2002), that such upregulation requires concomitant hippocampal activity (Ribeiro et al., 2002), and that it progresses over time in an anterograde manner across specific cortical areas (Ribeiro et al., 2002), we proposed that REM sleep is a privileged window for the propagation of memory traces from the hippocampus to the cerebral cortex (Pavlides and Ribeiro, 2003; Ribeiro and Nicolelis, 2004; Ribeiro et al., 2002).

While this model fulfills earlier psychological predictions of separate, sequential mnemonic roles for SWS and REM sleep (Giuditta, 1985; Stickgold, 1998), it is nonetheless controversial whether these states perform distinct mnemonic roles (Hirase et al., 2001; Kudrimoti et al., 1999; Louie and Wilson, 2001; Segawa, 2006; Sejnowski and Destexhe, 2000; Tononi and Cirelli, 2003). The mechanisms relating neural activity and IEG expression during sleep remain uncharted, and the model does not take into account the short intermediate sleep (IS) between SWS and REM sleep, a distinct state (Gottesmann, 1996) 
characterized by robust spindle oscillations that could in principle trigger IEG expression instead of REM sleep (Gervasoni et al., 2004). Recent results in humans show that the duration of SWS recorded a few hours after memory acquisition correlates positively with cortical engagement and hippocampal disengagement in memory encoding over the course of the following month, but REM sleep has not been assessed (Takashima et al., 2006). Finally, despite evidence of coordinated hippocampo-cortical interactions during sleep (Ji and Wilson, 2007; Qin et al., 1997; Siapas and Wilson, 1998; Sirota et al., 2003), electrophysiological data revealing hippocampofugal, corticopetal neural processing are still missing.

In the present study, the combination in the same animals of multielectrode recordings, online state detection, and in situ hybridization, allowed us to assess for the first time the relationship between firing rates, local field potentials (LFP), and plasticity-related IEG expression across the sleep-wake cycle. Exploration of novel objects in the dark was the experimental paradigm chosen to produce novel spatio-tactile stimulation (Ribeiro et al., 1999, 2004). The targeted brain areas comprised the hippocampus (HP), the primary somatosensory cortex (S1), and the primary visual cortex (V1). S1 and HP were chosen because of their direct involvement with tactile (Simons and Woolsey, 1979) and spatial (O'Keefe, 1979) processing, respectively. V1 was used as a negative control for non-specific, arousal-related neural changes. All three areas show persistent changes in neuronal activity during post-experience sleep (Ji and Wilson, 2007; Pavlides and Winson, 1989; Ribeiro et al., 2004), and respond with robust (IEG) activation during novel stimulation (Grimm and Tischmeyer, 1997; Ramirez-Amaya et al., 2005; Wallace et al., 1995). Here, we report evidence that each sleep state plays a distinct and complementary physiological role in memory consolidation. The data indicate that novel experience is followed by persistent cortical - but not hippocampal - plasticity during sleep.

\section{MATERIALS AND METHODS \\ Animals}

A total of 32 adult male rats (Long-Evans, $300-350 \mathrm{~g}$ ) were studied. Two separate groups of four and five rats were used for strictly behavioral experiments (object learning). Joint IEG expression and neural recordings were performed in 14 animals, additional 4 rats were only used for recordings, and additional 5 rats were only used for IEG expression assessment. In the latter case animals had their behaviors recorded, were kept awake, and served as negative and positive waking controls (WK- and WK+, respectively). WK- animals were kept awake for 2 hours and killed without exposure to novel experience. WK+ animals were kept awake for 2 hours, exposed to novel experience for 20 minutes and killed 10 minutes after experience offset. The SWS+, IS+, and REM+ groups were killed approximately 4 hours after experience. Note that the $W K+$ group differs from all the other $(+)$ groups with regard to post-experience survival times. Negative controls for REM sleep (REM-) were investigated for arc expression; results are shown in Figure S1 of Supplemental Data. Negative controls for other sleep states were not investigated due to the previous demonstration of zif- 268 downregulation during sleep states that are not preceded by novel stimulation (Pompeiano et al., 1994; Ribeiro et al., 1999, 2002). Altogether, the following groups were subjected to IEG assessment, depending on the reference state and the presence $(+)$ or absence $(-)$ of previous novel experience: REM- $(n=3)$, WK- $(n=3)$, WK+ $(n=3)$, SWS $+(n=3), \mathrm{IS}+(n=3)$, and REM $+(n=4)$. Housing, surgical, and recording procedures were in accordance with the National Institutes of Health guidelines, the Duke University Institutional Animal Care and Use Committee, and the ELS-IINN Committee for Ethics in Animal Experimentation.

\section{Novel object exploration}

Spatio-tactile novel stimulation was produced by transiently introducing four different objects in the corners of the recording box for 20 minutes, as previously described (Ribeiro et al., 2004). Exposure to novel objects elicits robust IEG transcriptional upregulation in the hippocampus and primary sensory cortices during WK (Wallace et al., 1995), and REM sleep (Ribeiro et al., 1999), but the recall of novel object memories becomes hippocampal-independent over time (Forwood et al., 2005; Gilbert and Kesner, 2004). All recordings were performed under infrared illumination. This prevented stimulation of $\mathrm{V} 1$, allowing us to distinguish between modality-specific and arousal-related effects on neuronal firing rates and IEG expression.

\section{Object learning tasks}

Two experiments modified from Mumby et al. (1995) were performed to assess object learning over 1 day and 1 week (results presented in Figures $1 \mathrm{~A}$ and $1 \mathrm{~B}$, respectively). In the first task (novelty-preference), animals $(n=5)$ were exposed to four novel objects for 20 minutes at midnight in the dark; 24 hours later the same animals were similarly reexposed to two of the same familiar objects (places unchanged), and two additional novel objects. Time spent in the exploration of novel objects was compared to time spent exploring familiar objects. In the second task (familiarity), animals $(n=4)$ were repeatedly exposed to the same objects (places unchanged) for 20 minutes at midnight for 7 consecutive days, and time spent in object exploration was measured.

\section{Multielectrode implantation}

The general approach for electrode implantation and multichannel recording has been described elsewhere (Kralik et al., 2001; Nicolelis et al., 2003; Ribeiro et al., 2004). Rats were anesthetized with ketamine and xylazine and implanted with three multielectrode arrays of tefloncoated tungsten microwires $(35 \mu \mathrm{m}, 1.0-1.2 \mathrm{M} \Omega$ at $1 \mathrm{kHz}$; California Fine Wire Company, Grover Beach, CA). Stainless steel screws and dental acrylic were used to secure the implant. One screw was soldered to a silver wire to serve as recording ground. The accuracy of electrode placement during surgery was assured by stereotaxic placement of microwires and by continuously recording neural activity during the surgery. Arrays targeting HP, S1, and V1 (Figure 1C) were centered on the following stereotaxic coordinates on the left hemisphere, in $\mathrm{mm}$ from Bregma with respect to the antero-posterior (AP), medio-lateral (ML), and dorso-ventral (DV) axes (Paxinos and Watson, 1997): HP (AP: -2.80; ML: +1.5; DV: -3.30 ); S1 (AP: -3.00 ; ML: +5.5; DV: -1.40 ); V1 (AP: -7.30 ; ML: +4.00; DV: -1.30 ). DV measurements were taken with respect to the pial surface. Arrays consisted of 16-32 microwires spaced at $250 \mu \mathrm{m}$ intervals $(4 \times 4$ arrays for S1 and V1, $2 \times 16$ array for HP) attached to plastic connectors (Omnetics, Minneapolis, MN). For S1 and V1, arrays were aimed at pyramidal layer V and the location of implants was confirmed by the respective presence of somatosensory or visual responses during implantation and/or experimentation. For HP, electrode placement was guided by the characteristic depth profile of ongoing neuronal activity during implantation, and by the presence of theta rhythm in LFPs observed during test recordings of alert WK and REM sleep.

\section{Electrophysiological recordings}

After 1-week recovery from surgery, animals were habituated to the empty recording box for 5 full consecutive days (12:12 light cycle, lights off at 7 PM, food and water ad libitum). Experiments consisted of recording animals before, during, and after object exploration in the dark (Figure 1D). On the day of the experiment, arrays were plugged to the recording cables under halothane anesthesia around 6:30 PM under normal illumination. Animals were then placed inside the dark recording chamber under infrared illumination. To avoid the residual effects of halothane, unit waveform selection began 60 minutes after placing the animals inside the recording box. Recordings usually begin at 9 PM. A 96-channel multineuron acquisition processor (MAP, Plexon Inc, Dallas, TX) was used for digital spike waveform discrimination and storage. Continuous single-unit recordings were performed for up to 6 hours using a software package for 


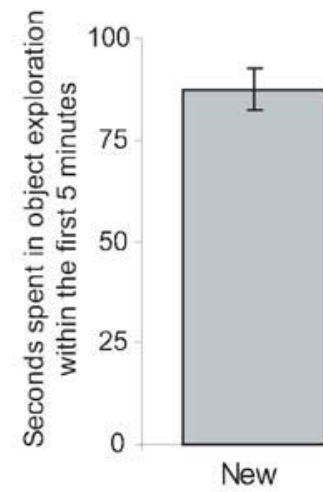

objects

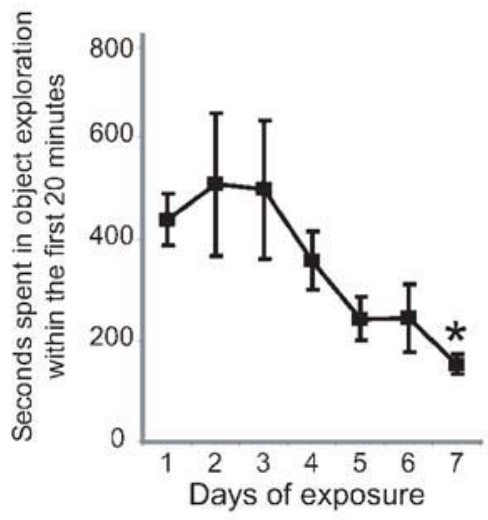

C

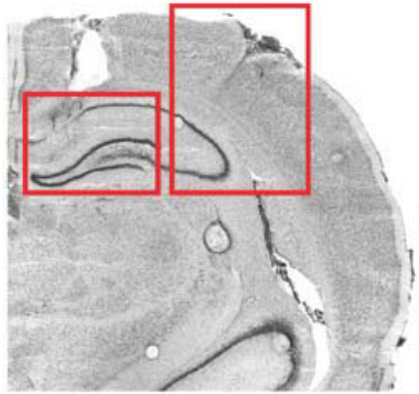

Anterior

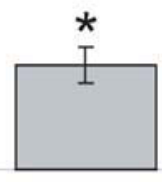

Familiar objects

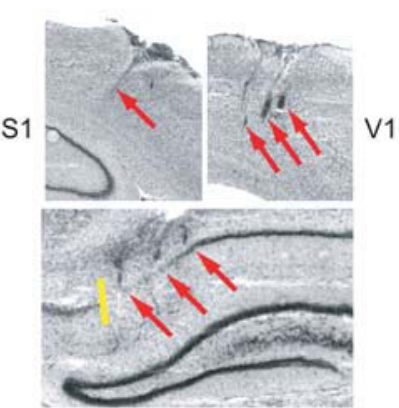

HP

D

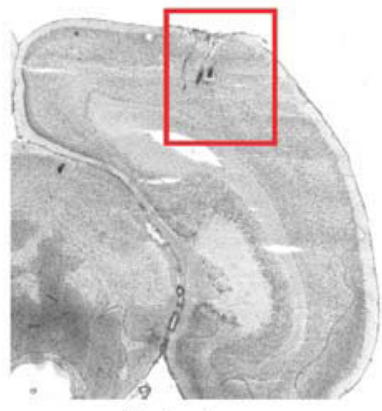

Posterior

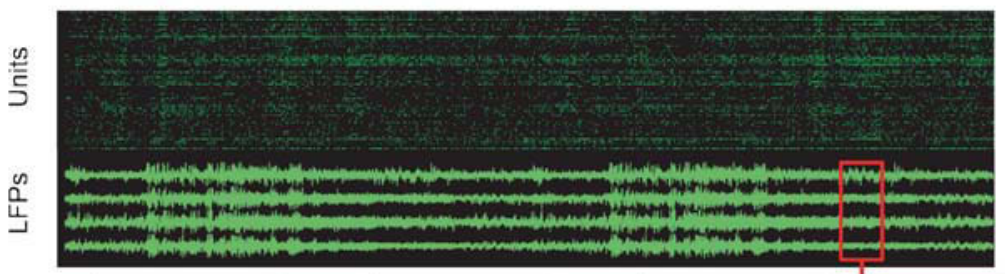

online state-mapping
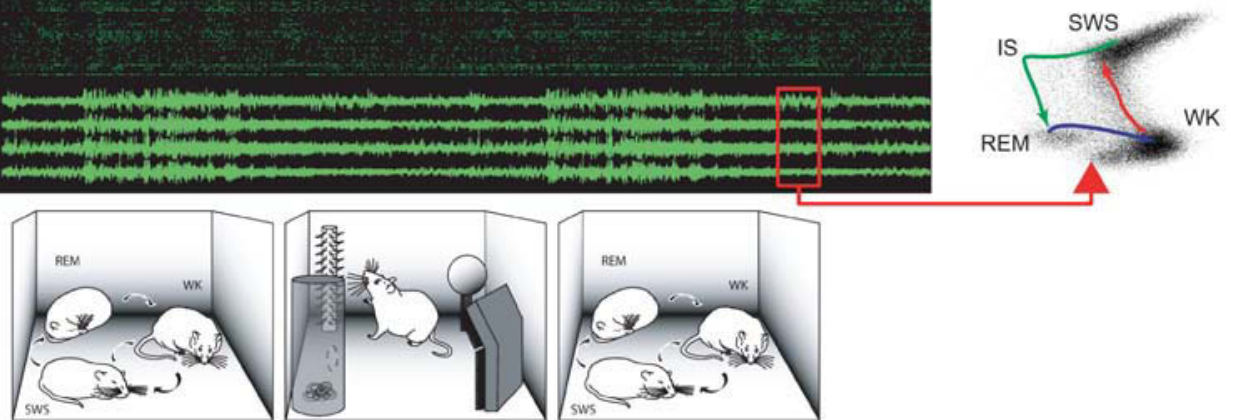

GROUPS

PRE-EXP (2h)
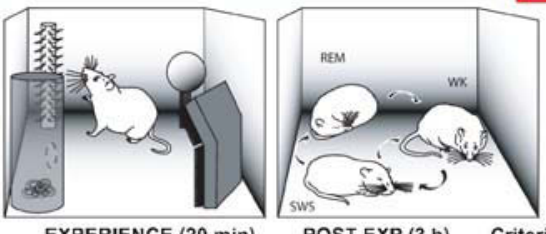

EXPERIENCE $(20 \mathrm{~min})$

POST-EXP (3h) Criteria (1 h)

in situ hybridization

REM (+) WK SWS IS REM
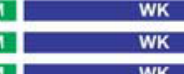

WK

WK

WK SWS IS REM REM

SWS (+) WK SWS IS REM

WK $(+)$ WK SWS IS REM

WK (-)

$\operatorname{REM}(-)$

WK SWS IS REM

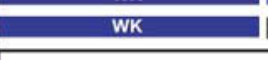

WK SWS IS REM NO REM

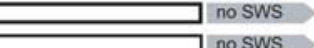

no sws

REM

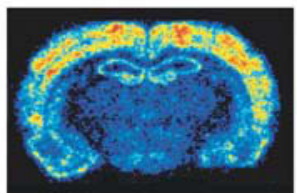

Figure 1. Experimental design to probe cortical and hippocampal changes following novel object exploration. (A) A novelty-preference task indicates object learning 1 day after a single exposure. Time spent in the exploration of novel objects within the first 5 minutes of exposure was significantly higher than time spent exploring familiar objects (means \pm SEM, paired $t$-test, $P=0.002$ ). (B) A familiarity task with seven consecutive daily exposures to the same objects reveals incremental object learning. Shown is the progressive reduction of time spent in object exploration, as animals habituate to the stimuli (means \pm SEM, Anova $F(6,21)=2.679, P=0.04$, Fisher's $P L S D P=0.027$ ). (C) Anatomical location of implants on cresyl-stained brain sections. Electrode tracks indicated by red arrows. Yellow line marks the CA1/subiculum border. (D) Experiments comprised up to 6 hours of recordings under infrared illumination (cables omitted; top panel in green and black represents unit and LFP recordings). Novel objects were introduced in the box for 20 minutes. Three hours after object removal animals began to be monitored for 1 more hour, being prevented to progress into specific sleep-wake states of interest according to their randomly assigned group. Animals were killed 30 minutes after reaching state criteria (see Materials and Methods), and brains were processed for arc and zif-268 expression. WK and REM controls without (-) stimulation were also studied; WK (+) animals were killed 10 minutes after object removal. Therefore, the WK (+) condition represents an immediate post-exposure time point, rather than a period of post-exposure wake similar in post-exposure time to the other (+) conditions. Note that REM (+) animals were the only ones that were allowed uninterrupted sleep in the last hour of the experiment. 
supervised spike sorting which allows for real-time sampling of all spike waveforms (SortClient 2002, Plexon Inc) for offline validation (Offline Sorter 2.3, Plexon Inc) (Figure S2 of Supplemental Data). LFPs recorded from the same wires were pre-amplified (500X), filtered $(0.3-400 \mathrm{~Hz})$, and digitized at $500 \mathrm{~Hz}$ using a Digital Acquisition card (National Instruments, Austin, TX) and a MAP (Plexon). The LFP frequencies actually analyzed in this study ranged from 1 to $55 \mathrm{~Hz}$. Behaviors were recorded throughout the entire experiment under infrared illumination, by way of two CCD video cameras and a videocassette recorder. Video and neural recordings were synchronized with a millisecond-precision timer (model VTG-55; For-A, Tokyo, Japan). Staggered arrays were used to simultaneously record neurons from the CA1 field and LFPs from the DG, due to its very robust theta rhythm. About $80 \%$ of the electrodes were targeted to CA1. These electrodes were cut $0.8 \mathrm{~mm}$ shorter than the longer electrodes, destined to the DG. Analysis of our data (Bonferroni $t$-test, $P<0.05$ ) showed that only $21 \%$ of $D G$ neurons were activated by experience, and were pooled with activated CA1 neurons. Therefore, hippocampal data mostly reflect CA1 neurons ( $>90 \%$ of total HP neurons).

\section{Histology}

Neuroanatomical reconstruction to confirm multielectrode placement and guide IEG expression quantification was carried out after experiments were finished, by inspecting $20 \mu \mathrm{m}$ cresyl-stained frontal brain sections with reference to anatomical planes (Paxinos and Watson, 1997). To prevent interference with IEG detection, lesion holes were not employed for anatomical reference. Instead, glial scars along electrode tracks were used to verify the correct placement of the recording arrays. Inspection showed that electrodes targeting the HP were never placed in the subiculum, which at the antero-posterior level targeted appears only in the most medial $0.5 \mathrm{~mm}$ of the brain hemispheres (Figure 1C).

\section{Behavioral state sorting}

At the end of 3 hours of post-experience recordings with free sleep-wake cycles, animals were monitored for an additional hour in which they were either selectively prevented from entering a specific sleep state or were let free to cycle through all states, depending on which group they were randomly assigned to. State prevention was achieved by systematically waking animals up (by gently tapping the recording box) before they transited into target states, as determined by a 1-second lag online state detection system (see below). Thus, animals randomly assigned to the WK group were prevented to sleep, animals in the SWS group were repeatedly woken up before transiting into IS, IS animals were woken up before entering REM sleep, and animals in the REM group were allowed to sleep freely. After 30 minutes of state prevention, animals were monitored for the occurrence of a criterion amount of the sleep state immediately preceding the excluded state ( 5 minutes for SWS, 15 seconds for IS, and 30 seconds for REM sleep, as total time spent in state within the past 30 minutes). Once this criterion was achieved, animals were monitored for an additional 30 minutes to reach maximum arc/zif-268 mRNA levels with reference to the moment when criterion was reached. The reason for waiting 30 minutes after reaching state criterion was the induction kinetics of arc and zif- $268 \mathrm{mRNA}$, characterized by a bell-shaped profile that peaks 30 minutes after a reference state or stimulus, investigated at intervals of 5, 10, or 15 minutes (Guzowski et al., 1999, 2001; Mello and Clayton, 1994). Our experimental design prevented the occurrence of the excluded state in the entire 60 minutes that preceded killing. By the same token, it enriched the amount of the preceding state around 30 minutes before killing. This strategy allowed us to cope with the slow dynamics of mRNA transcription, enabling for effective state "dissection" in the context of a highly inertial process such as IEG transcription. Figure 7 shows the state dynamics obtained with this method in each animal used for the assessment of state-dependent IEG expression. For instance, consider rat \# 12: the animal was awake from 60 to $~ 38$ minutes prior to killing, had several episodes of SWS and IS until 20 minutes prior to killing, and then stayed awake for the remaining time except for a brief SWS episode $\sim 16$ minutes prior to killing. Mean state amounts per hour were stable over recording time (Figure S3 of Supplemental Data), except for the last hour of recording in which the sleep-wake architecture was perturbed to sort animals in different groups. Note that the REM sleep episodes investigated occurred 4 hours after novel experience, with free sleep-wake cycles in between. In average, this resulted in the investigation of the 28th REM sleep episode after novel experience in the REM (+) group.

\section{Online state detection}

To sort sleep-wake states in the last hour of survival of animals destined to IEG expression analysis, we used a previously described quantitative spectral algorithm for behavioral state classification based on hippocampal and cortical LFPs (Gervasoni et al., 2004). Briefly, a two-dimensional state space was defined by two spectral amplitude ratios calculated by dividing integrated spectral amplitudes at selected frequency bands from LFPs simultaneously recorded in the areas of interest. A scatter plot of the two chosen LFP spectral amplitude ratios (state-space) reveals distinct clusters that correspond to different sleep-wake states (Figure S4 of Supplemental Data). This representation allowed us to monitor withinand between-state trajectories with a lag of 1 second, dissecting apart all the states of interest nearly in real time (Figure 1D, top-left panel and Figure S5 of Supplemental Data). An offline version of the same algorithm was used to sort behavioral states throughout experiments for all other analytic purposes.

\section{In situ hybridization}

At the end of recordings, animals were quickly placed inside a lightproof halothane chamber, anesthetized, and decapitated for quick brain extraction and freezing in a mixture of dry ice and ethanol. Brains were stored at $-70^{\circ} \mathrm{C}$ until sectioning. Serial frontal sections $(10 \mu \mathrm{m})$ were processed by in situ hybridization with ${ }^{35} \mathrm{~S}$-labeled riboprobes $(1.5 \mathrm{kB}$ for Arc, $3 \mathrm{kB}$ for Zif- 268) according to a previously described protocol (Clayton et al., 1988) to assess IEG mRNA levels (Figure 1D, bottom-right panel; see Figure S6 of Supplemental Data). Sections were then dried, exposed to a high-resolution phosphor screen for 48 hours, and scanned on a Storm Phosphorimager system (Amersham Biosciences, Sunnyvale, $\mathrm{CA})$. Absence of sense-strand hybridization was used as a control for signal specificity. Multiple hybridizations (five for Zif-268, two for Arc) were performed with equivalent sets of sections, to optimize stringency conditions and to assess consistency of results across different batches.

\section{IEG expression quantification}

Blind phosphorimager densitometric quantifications were performed with ImageQuant 5.0 software (Amersham Biosciences). Arc and zif-268 levels were measured on both hemispheres to control for implant-related changes in IEG expression; no significant differences were found across hemispheres. Mean IEG expression level for each animal was calculated (in arbitrary phosphorimager counts with background subtraction) as the average of IEG expression levels sampled from both hemispheres of six different brain sections, spanning $\sim 800 \mu \mathrm{m}$ of tissue for each brain region of interest. HP measurements were restricted to the CA1 field, where most of the hippocampal units were recorded from. In S1 and V1, measurements were taken as a rectangular slab across all layers (zif-268) or restricted to layers $\| / / I I$ and $\mathrm{V}(\operatorname{arc})$.

\section{Normalization and statistics}

Firing rate normalization in Figures 2 and $3 \mathrm{C}$ was performed separately for each neuron, so that all time bins were normalized by the minimum ( $\min$ ) and maximum (max) firing rates found within the time interval of interest. The following equation was used to bind the normalized values between 0 and 1 : Xnorm $=(x-\min ) /(\max -\min )$. Normalization with reference to V1 (obtained by dividing $\mathrm{S} 1$ and $\mathrm{HP}$ measurements by corresponding V1 values) was used in Figures $4 B-4 D$ to remove non-specific 
arousal effects from IEG expression, firing rates and LFP amplitudes at specific frequency bands. V1 normalization was not employed in Figure 5 because the correlations in question had opposite profiles between cortical and hippocampal regions: while $\mathrm{HP} \rightarrow \mathrm{S} 1$ and $\mathrm{HP} \rightarrow \mathrm{V} 1$ showed a monotonic increase in WK/EXP $\rightarrow$ REM/POST correlations, S1 $\rightarrow$ HP and $\mathrm{V} 1 \rightarrow \mathrm{HP}$ showed monotonic decrease. The use of V1- normalization would in this case distort the contrast between hippocampal and cortical regions. Since firing rates were approximately normally distributed across all groups as determined by the Kolmogorov-Smirnov test, parametric statistics were used for the analysis of firing rates (ANOVA, $t$-test). A Pearson correlation analysis across animals was used in Figure 5, in which each data point used to estimate $R$ represented measures from one animal. Uncorrected degrees of freedom were employed because the datasets derived from repeated measures taken at successive points in time (raw and V1-normalized firing rates and LFP amplitudes at specific frequency ranges) satisfied the sphericity assumption with $P<0.001$ (Bartlett's Sphericity Test). Deviation from normality was larger for LFP amplitudes and IEG expression levels, so these measurements were submitted to non-parametric statistical analyses (Kruskal-Wallis test followed by Mann-Whitney tests, and Spearman correlation). Bonferroni correction for multiple comparisons was calculated by multiplying the raw $P$ values by the number of comparisons effected in each case, as follows: five comparisons for EXP versus Post/Pre in Figure 3A (WK); six comparisons for Post versus Pre in Figure 3A (WK, SWS, and REM); ten comparisons for Post versus Pre in Figure 3B; two comparisons per gene in Figure 4C; two comparisons per frequency range per gene in Figure 4D; nine comparisons per panel in Figure 5.

\section{RESULTS}

Exploration of novel objects produces learning after a single exposure (Figure 1A), but repeated daily exposure to the same objects continues to produce learning over time (Figure 1B). A single 20 minute exposure to novel objects produces intense spatio-tactile stimulation, causing substantial changes in neuronal firing rates in all recorded areas (Figure 2). In 15 animals analyzed during object exposure, a total of 450 neurons were recorded in S1, 353 neurons in HP, and 320 neurons in V1. To identify neurons modulated by experience, we compared the mean firing rates of WK periods recorded during and immediately before experience. The mean proportions of neurons showing increased firing rates during experience were $57 \%$ in $\mathrm{S} 1,44 \%$ in $\mathrm{HP}$, and $34 \%$ in V1 (Bonferroni $t$-tests between WK/EXP and WK/Pre periods of 20 minutes duration, $P<0.01$ ). A smaller proportion of neurons showed significant firing rate decrease during experience $(9 \%$ in $\mathrm{S} 1,9 \%$ in $\mathrm{HP}, 15 \%$ in $\mathrm{V} 1)$. The relatively high percentage of V1 neurons with increased firing rates in the absence of visual stimuli was attributed to non-specific arousal (but see Discussion). The 'increase," "decrease," and 'ùnchanged" groups of neurons showed similar mean firing rates $(8.35 \pm 0.33,7.87 \pm 0.56$, and $7.67 \pm 0.38$; mean $\pm S E M$, respectively). The distributions of firing rates were unimodal, showed no significant differences across groups (Kolmogorov-Smirnov, $P>0.2$ ), and were characterized by a clear predominance of slow-spiking neurons (mode between 2 and $6 \mathrm{~Hz}$, with $70.3,81.1$, and $79.7 \%$ of cells below $10 \mathrm{~Hz}$ in the "increase," "decrease" and 'ùnchanged" groups, respectively). To the extent that principal neurons with excitatory function tend to fire less than inhibitory interneurons (Swadlow, 2003), and since our electrode implants were aimed at pyramidal layers in the hippocampus (CA1 field) and cerebral cortex (layer V), the results suggest that most of the recorded units were principal neurons. All subsequent analyses focused on activated neurons, i.e., units that showed increased firing rates during object exposure. No significant effects were found for non-activated or deactivated neurons.

\section{Neuronal reactivation in the hippocampo-cortical loop}

Next, we compared the mean firing rates of activated neurons in S1, HP, and V1 across WK, SWS, and REM sleep occurring between 2 hours preand 3 hours post-experience (respectively $n=9$, 8 , and 5 rats in which state amounts were abundant and comparable on an hourly basis, pre- and post-experience; state amounts in the remaining animals were variable over time and consequently were not useful for the purpose of a quantitative comparison). As expected, significant increases were detected during WK experience (EXP) in all three areas (Figure 3A, left panel; Anova HP_WK $\left.\left.F_{5 / 45}\right)=8.289 P=0.0001 ; S 1 \_W K F_{(5 / 45}\right)=3.792 P=0.006$; V1_WK $F(5 / 40)=6.251 P=0.0002$; (Bonferroni $t$-test WK_EXP > Pre1, Pre2, Post2, Post3 with $P<0.01$ ). In HP and V1, increases in WK firing rates persisted well into the first post-experience hour (Bonferroni $t$-test, $P<0.05$ ), while $S 1$ showed a similar but non-significant trend (Figure $3 \mathrm{~A}$, left panel). Increased firing rates after experience were observed during both SWS and

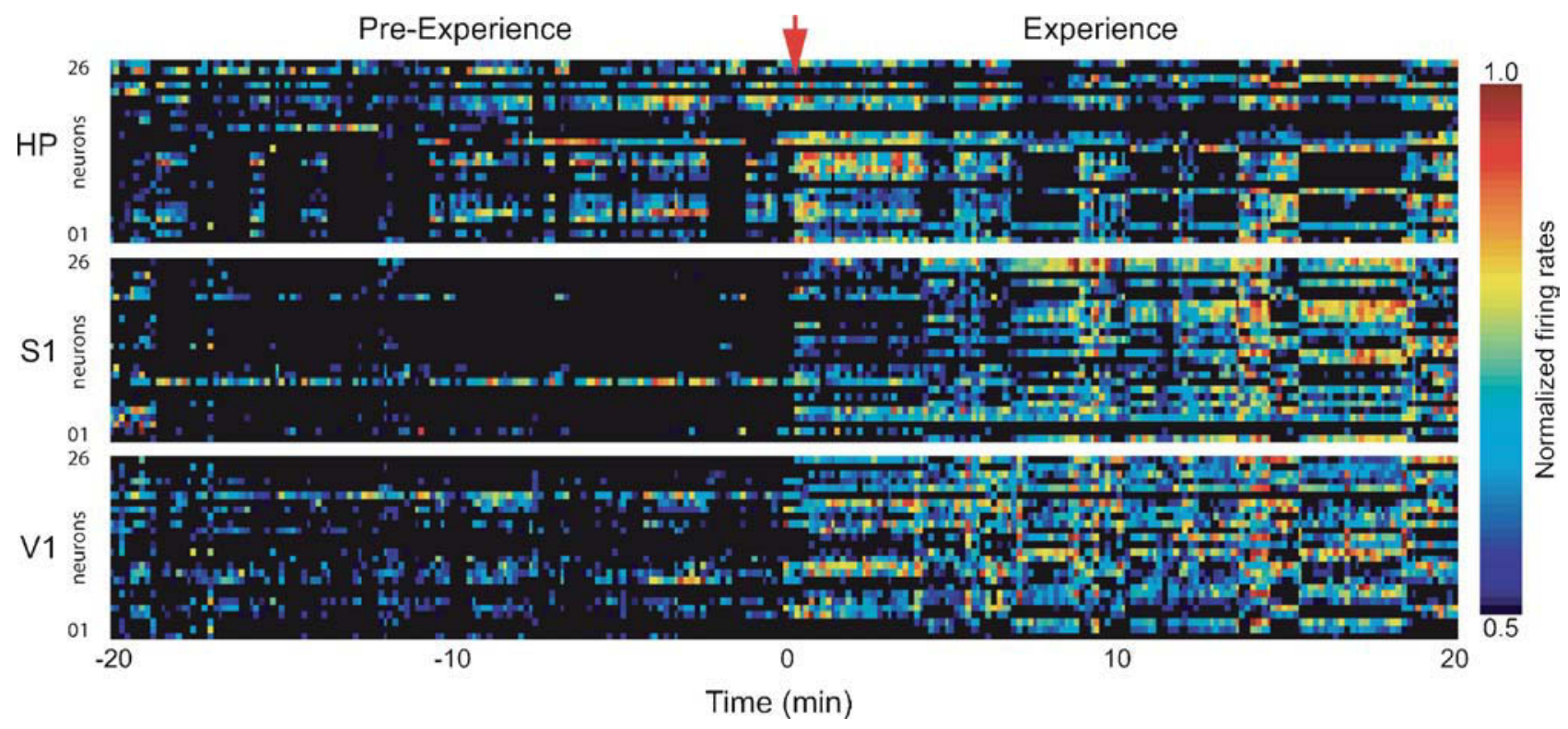

Figure 2. Firing rate changes during novel object exploration. Exposure to novel objects in the dark caused widespread changes in neuronal activity that persisted throughout the 20 minutes spatio-tactile experience. Shown are normalized neuronal firing rates for neurons in HP, S1, and V1 in a representative animal. Time $=0$ marks experience onset. 
A

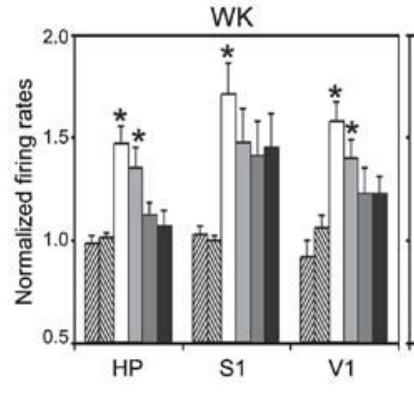

sws

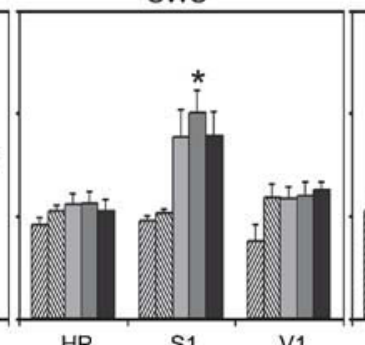

S1
REM

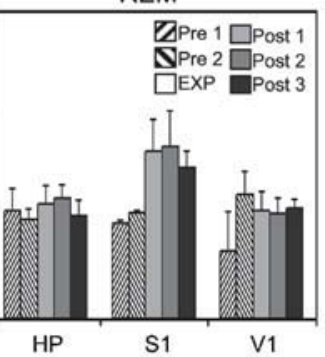

B

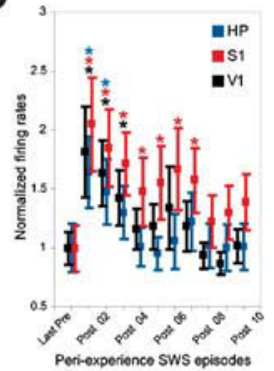

$\mathrm{C}$

EXP Post-Experience
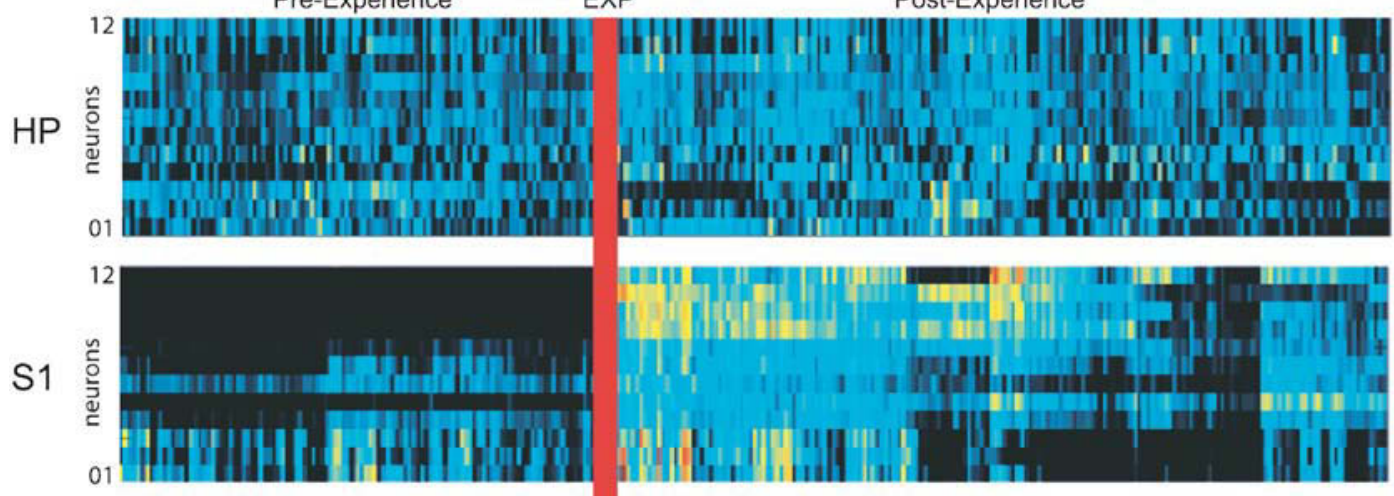

01

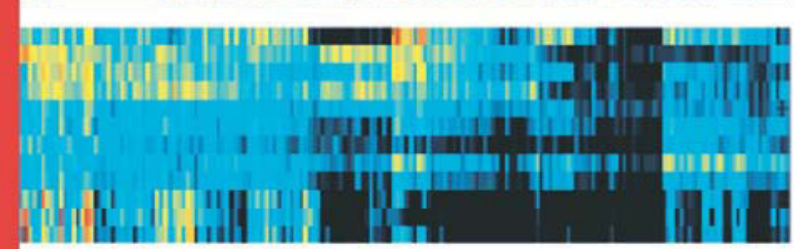

V1

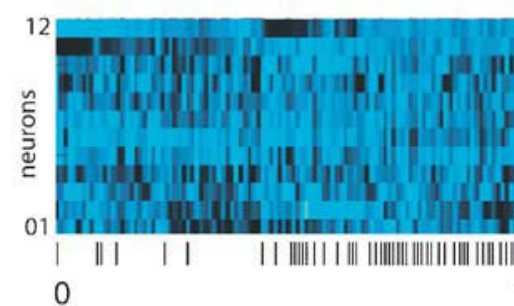

Time boundaries of concatenated SWS episodes (minutes)

Figure 3. Firing rate changes after novel object exploration. Long-lasting firing rate increases after novel spatio-tactile stimulation occurred during SWS in S1, but not in HP or V1. Asterisks indicate $P<0.05$ (Bonferroni t-tests). (A) Hourly evolution of neuronal activity across recorded areas and states (group data). Significant increase was detected during exposure in all recorded areas (EXP), with a gradual decay thereafter (Post 1). Rate increase reached robust significance during sleep only for SWS in S1 (Post 2), with a non-significant trend for Post 1 and Post 3 during SWS, and all Post hours during REM sleep. (B) Transient firing rate increase occurred in all recorded areas during the first few SWS episodes of the first hour following novel stimulation, with a marked decay within 20 minutes of stimulation offset. (C) Increased neuronal activity persisted for hours after experience offset during SWS in S1. Shown are the normalized firing rates during concatenated SWS episodes spanning an entire representative experiment. Ticks at the bottom indicate SWS episode boundaries.

REM sleep, but only during SWS recorded in the second post-experience hour the effect reached robust statistical significance (Figure 3A, middle and right panels; Anova S1_SWS $\left.F_{(4 / 37}\right)=5.463 P=0.0015$; S1_REM $F(4 / 22)=1.768 P=0.1714$; Bonferroni $t$-test SWS_Post2 $>$ Pre1, Pre2 with $P<0.05)$. $S 1$ firing rates during SWS also showed an elevation trend in the first and third post-experience hours (Bonferroni $t$-test SWS_Post1 > Pre1, Pre2 with $P=0.12$ and SWS_Post $3>$ Pre1, Pre2 with $P=0.1$ ). No such post-experience changes were seen in HP and V1 (HP_SWS $\left.F_{(4 / 37}\right)=0.585$ $P=0.6755$; HP_REM $F(4 / 22)=0.346 P=0.8439$; V1_SWS $\left.F_{(4 / 33}\right)=2.294$ $P=0.0802$, V1_REM $F(4 / 18)=0.805 P=0.5380$ ). To have a more comprehensive view of the effects of object exposure on post-experience firing rates, we collapsed the data across pre- and post-exposure hours and performed simple pre-versus post-paired $t$-tests. Using this less stringent comparison, we found significant firing rates increases in the following areas and states: WK_HP $(P=0.043)$, WK_V1 $(P=0.004)$, SWS_S1 $(P=0.001)$, SWS_V1 $(P=0.04)$, REM_S1 $(P=0.05)$.

Previous studies of hippocampal reactivation during SWS (Pavlides and Winson, 1989; Wilson and McNaughton, 1994) reported firing rate changes as short-lived as 20 minutes, so we also investigated firing rates during the initial SWS episodes following object removal. All recorded areas showed strong post-experience activation in the first SWS episode after object exploration, with a steep decay to basal levels of activity after a few SWS episodes within the first post-experience hour (Figure $3 \mathrm{~B}$; note the persistence gradient $\mathrm{HP}<\mathrm{V} 1<\mathrm{S} 1$ ). The rate profile of individual neurons during SWS in a representative animal illustrates the progression of post-experience persistent changes in neuronal activity (Figure 3C). The data indicate that the generalized surge of neuronal activity initially caused by novel experience is followed by long-lasting firing rate changes that are maximal during SWS and specific to the S1 cortex.

\section{Sleep-dependent IEG expression}

We then investigated the neural correlates, state-dependency, and hippocampo-cortical distribution of arc and zif-268gene expression before and after novel experience. Arc expression levels were correlated to zif268 levels in HP (Rho $=0.475, P=0.05)$ but not in $\mathrm{S} 1(\mathrm{Rho}=0.333$, $P=0.169$ ) or V1 (Rho $=0.22, P=0.365$ ). Same IEG correlations across areas were positive and significant for all but two of the combinations 
tested (arc S1 vs. V1 Rho $=0.622, P=0.01 ; \operatorname{arc} \mathrm{S} 1$ vs. HP Rho $=0.447$, $P=0.0654 ;$ arc HP vs. V1 Rho $=0.730, P=0.003 ;$ zif-268 S1 vs. V1 Rho $=0.236, P=0.33 ;$ zif-268 S1 vs. HP Rho $=0.756, P=0.002 ;$ zif-268 HP vs. V1 Rho $=0.6, P=0.0134)$. These results reveal moderate coupling of IEG expression across areas, partially reflecting the non-specific increases in neuronal activity caused by the enhanced arousal reflected in V1changes.
As shown in representative autoradiograms (Figure 4A), arc and zif268 gene expression were strongly upregulated in the S1, V1, and HP during novel object exploration (Figure 4A, compare WK (-) versus WK $(+)$ in first and second columns). During post-experience SWS and IS, IEG levels were low throughout the hippocampo-cortical circuit (Figure 4A, third and fourth columns). Most importantly, IEG expression was reinduced in S1 during REM sleep, but not in V1 or HP (Figure 4A, fifth column).

A

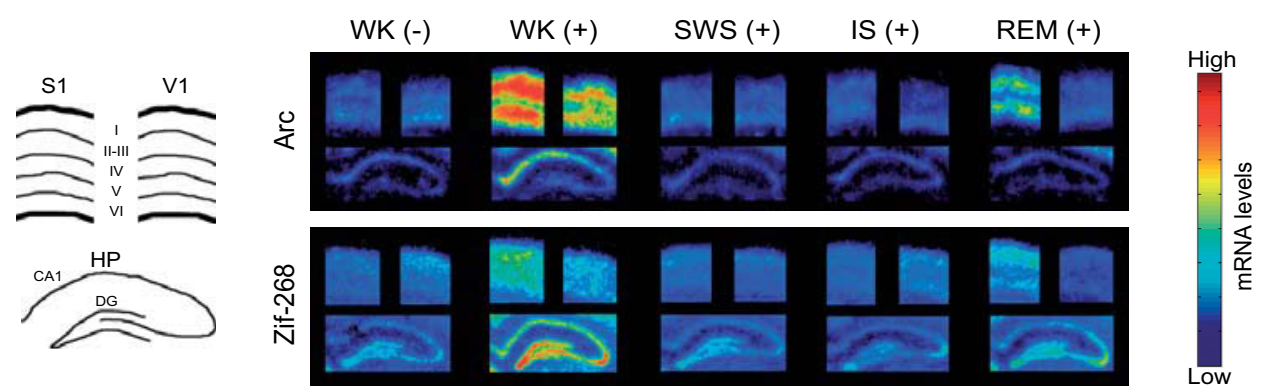

B
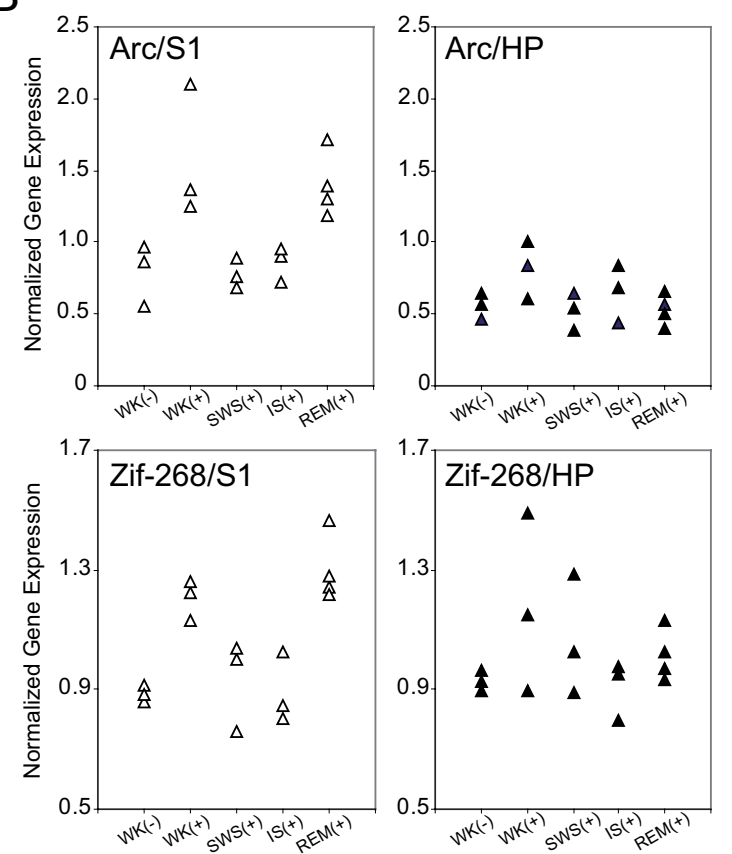

C

\section{Firing Rates}

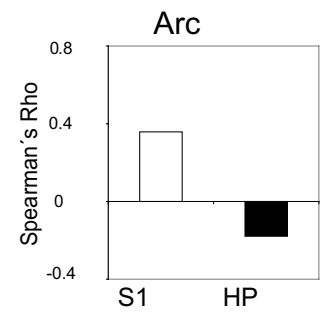

D

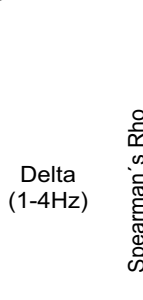

Local Field Potentials
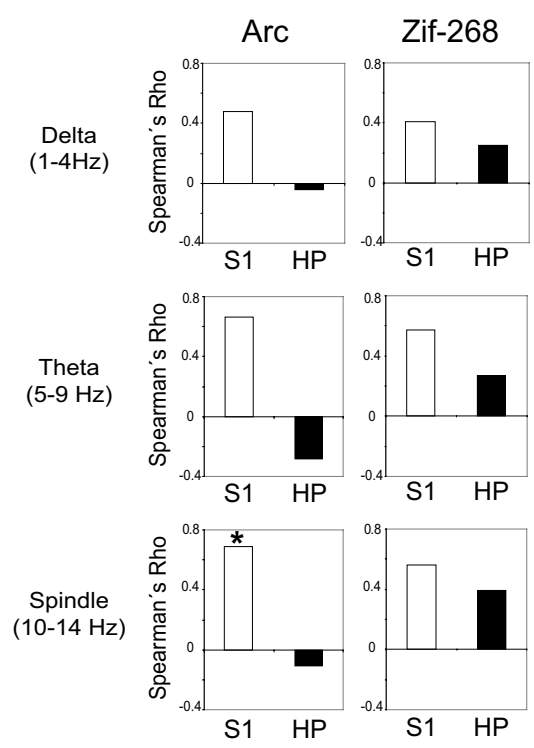

Beta

$(15-22 \mathrm{~Hz})$
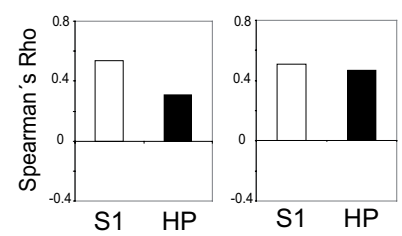

Gamma $(25-55 \mathrm{~Hz})$
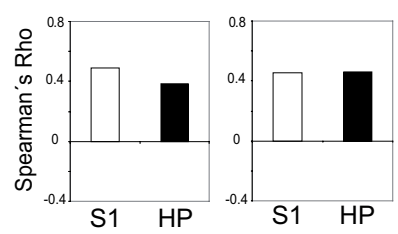

Figure 4. Neural correlates of state-dependent IEG expression in the hippocampo-cortical loop. (A) Autoradiograms of representative in situ hybridizations show that novel spatio-tactile stimulation induced IEG upregulation in S1, HP, and V1 during experience. Post-experience IEG reinduction was mostly localized to pyramidal layers in S1, and occurred specifically during REM sleep. Drawing on the left indicates cortical layers and hippocampal regions dentate gyrus (DG) and CA1 field. (B) Data points normalized by V1 levels show IEG upregulation during WK (+) and REM sleep in S1 (P<0.05), but not in HP. (C) Spearman correlations between V1-normalized IEG expression and firing rates were not significant. (D) Spearman correlations between V1-normalized IEG expression and LFP amplitudes in different frequency ranges were higher in S1 than HP $(P<0.01)$. There is a significant correlation between spindle-range LFP oscillations and arc expression in $S 1$ (asterisk, $P<0.05$ ), but not in $H P$. 
To disambiguate the dynamics of IEG expression in the hippocampocortical loop from arousal effects, S1 and HP expression levels were normalized by V1 measurements within each animal (see Materials and Methods; non-normalized IEG data shown in Figure S7 of Supplemental Data). The V1-normalized group data show that arc and zif-268 were significantly upregulated in S1 but not in HP (Figure 4B; Kruskal-Wallis test, $\mathrm{DF}=4$ : for $\operatorname{Arc}, H=11.603, P=0.0206$ in $\mathrm{S} 1$ and $H=5.702, P=0.23$ in HP; for Zif-268, $H=11.338, P=0.023$ in $\mathrm{S} 1$ and $H=3.382, P=0.50$ in $\mathrm{HP})$. For both IEGs, normalized expression levels in S1 were significantly higher than pre-experience WK immediately after novel experience (WK+) and during post-experience REM sleep ( $P<0.05$ by Mann-Whitney). In contrast, normalized IEG expression in S1 did not differ significantly between pre-experience WK and post-experience SWS or IS. Normalized IEG expression levels in HP did not vary significantly across states. Despite the link between cortical IEG upregulation and post-experience REM sleep, analysis of the relation between IEG expression in S1 and time spent in REM sleep within 60 minutes before killing showed no significant correlation (arc Rho $=0.408, P=0.0924$; zif-268 Rho $=0.335$, $P=0.1674)$.

To investigate the neural correlates of IEG expression in S1 and HP, we estimated the Spearman correlations across animals between IEG expression levels and mean firing rates (both normalized by V1 levels). IEG expression was not significantly correlated with firing rates in either
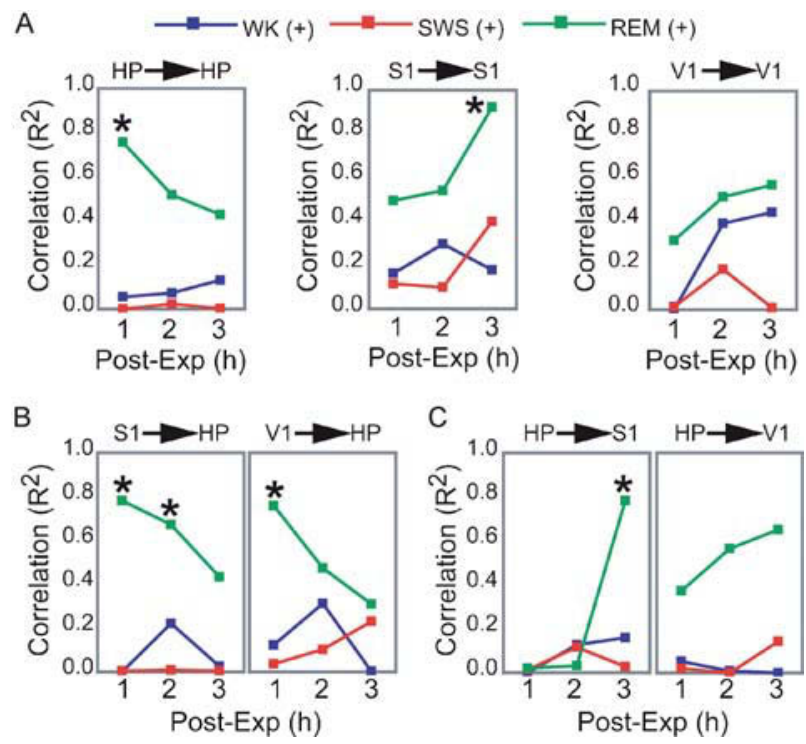

Figure 5. Dynamics of hippocampo-cortical activation after novel object exploration. (A, B, C) Pearson correlations between neuronal activity changes during experience and post-experience hours, within and across brain areas, were calculated across all animals based on a grand average of firing rates per hour per state (i.e., each data point used to estimate $R$ represents measures from one animal within a given state and post-experience hour; all values normalized by pre-experience levels; asterisk indicates $P<0.05$, Bonferroni t-tests; for state amounts over time, see Figure S3 of Supplemental Data). Significant state-sorted correlations were only detected during REM sleep and were all positive. While HP activation during experience could only predict HP activation in the first post-experience hour, $S 1$ activation during experience was poorly predictive of $\mathrm{S1}$ activity in the first two post-experience hours and highly predictive in the third hour. HP activation during experience was also progressively more predictive of post-experience $S 1$ activation over time. Conversely, S1 activation during experience predicted HP post-experience activity in a decaying manner over time. V1 showed a trend similar to S1, but weaker. These results provide electrophysiological support to the notion of cortical engagement and hippocampal disengagement during post-experience REM sleep, previously suggested by IEG expression experiments (Ribeiro et al., 2002). area (Figure 4C). We also assessed the Spearman correlations between V1-normalized IEG expression and amplitude of LFP oscillations within physiologically relevant frequency bands (Figure 4D). A significant positive correlation between arc expression and spindle-range LFP activity was observed in $\mathrm{S} 1$ but not in HP $(P<0.05)$. Furthermore, $\mathrm{S} 1$ correlations across all LFP frequency bands were significantly stronger than those in HP for arc (Mann-Whitney of Rho values, $P=0.009$ ). A similar trend was seen for zif-268, without, however, reaching criterion for statistical significance (Mann-Whitney of Rho values, $P=0.076$ ).

\section{Hippocampo-cortical dynamics after novel experience}

To further assess the state-dependency of post-experience neuronal processing in HP and S1, we asked whether the degree of firing rate increase produced by novel experience in a given brain area could explain post-experience neuronal activity changes observed in the same or another area. For that, we calculated the hourly evolution of Pearson correlations between experience and post-experience rate modulations according to brain area and state (only excitatory responses were considered; same animals as in Figure $3 A$; see Materials and Methods).

Significant correlations were only observed in REM sleep, all positive (Figures 5A-5C). Within-area correlations showed opposite dynamics for HP and S1: hippocampal rate modulation during experience predicted persistent HP changes only in the first post-experience hour (Figure $\left.5 \mathrm{~A}, R^{2}=0.755\right)$. In contrast, $\mathrm{S} 1$ correlations increased sharply from the first to the third post-experience hour, indicating that although cortical rate modulation during experience is not predictive of subsequent cortical changes in the short-term, it is highly predictive in the long-term $\left(R^{2}=0.923\right)$. The most intriguing results came from an assessment of Pearson correlations across the hippocampo-cortical axis. S1 changes during experience were strongly proportional to hippocampal activity in the first and second post-experience hours $\left(R^{2}=0.781\right.$ and 0.673$)$, decaying thereafter (Figure 5B). On the other hand, hippocampal changes during experience were poorly predictive of cortical changes in the first two post-experience hours, but very high correlations with $\mathrm{S} 1$ firing rates appeared during REM sleep in the third hour after experience $\left(R^{2}=0.783\right.$; Figure 5C).

\section{DISCUSSION}

In this study, we found that increases in the neuronal activity evoked by novel spatio-tactile stimulation faded within minutes in the HP or V1, but lasted for hours in S1 during sleep. Post-experience reactivation of neuronal firing in S1 peaked during SWS (Figure 3), while IEG expression linked to synaptic remodeling was specifically upregulated during post-stimulation REM sleep episodes (Figures 4A and 4B). Immediately after experience, IEG upregulation occurred in both the hippocampus and the cortex, but subsequent reinduction triggered by REM sleep was only cortical. LFP oscillations 1 hour prior to killing were proportional to arc expression in S1 but not in HP, particularly in the $10-14 \mathrm{~Hz}$ frequency range that characterizes cortical sleep spindles (Figure 3D). Importantly, cortical firing rate changes during REM sleep 3 hours after experience were proportional to hippocampal changes during experience, but not the converse (Figures $5 \mathrm{~A}-5 \mathrm{C}$ ). This asymmetry was possible because in each comparison two different moments in time were compared, i.e., hippocampal changes during experience were proportional to cortical changes 3 hours after experience, but cortical changes during experience were not proportional to hippocampal changes 3 hours after experience.

As previously noted for WK conditions (Guzowski et al., 2001), the range of arc expression upregulation during REM sleep was twice as large as that of zif-268 $(50-60 \%$ vs. $20-30 \%$, respectively, Figures $4 \mathrm{~A}$ and 4B), a difference that fits a direct role of the arc protein on morphological plasticity (Lyford et al., 1995) as well as the indirect, transcriptional role played by the zif-268 protein (Thiel et al., 1994; Wisden et al., 1990). ArC gene expression in the cortex showed correlation with LFP amplitude in the 
spindle-range (10-14 Hz) but not with firing rates (Figures 4C and 4D), as expected from signals more related to dendritic input than to somatic output (Lopes da Silva, 1991; Nunez, 1981; Thiel et al., 1994; Wisden, 1990). Indeed, arc mRNAs localize to dendrites in an activity-dependent manner (Steward et al., 1998). The correlation between arc mRNA levels and LFP amplitude in the spindle-range suggests that arc expression may be proportional to specific parts of the fMRI BOLD signal, which is also strongly proportional to LFP amplitudes (Logothetis et al., 2001; Niessing et al., 2005). This observation has potential relevance for studies of human cognition.

In so far as the preference for novel objects reflects the consolidation of memories for the familiar objects, our stimulation paradigm can be said to induce learning, because a single exposure to novel objects (Figure $1 \mathrm{~A}$ ) is sufficient to render these objects familiar after a 1 day retention interval, as expected from previous studies (Mumby et al., 2005). On the other hand, repeated daily exposure to the same objects produces a progressive decrease in exploratory behavior (Figure 1B), suggesting that object exposure generates strong arousal effects that only subside after a longer period of memory maturation takes place. The design of our invasive experiments did not allow, however, for a direct comparison of neural changes and memory formation, since animals were killed a few hours after a single exposure to the novel objects, before learning could be assessed. The methodology employed was also limited by the fact that in situ hybridization assesses transcriptional changes but not translation of the final protein product. It is always possible that changes in mRNA levels do not cause equivalent changes in protein levels, although the previous studies show a tight correlation between mRNA and protein levels for zif268 (Wisden et al., 1990) and arc (Lyford et al., 1995; Ramirez-Amaya et al., 2005). Indeed, IEG reinduction during REM sleep may explain the second wave of arc protein expression detected 8-24 hour after a single exploration session (Ramirez-Amaya et al., 2005). Another potential limitation of our study is the arousal confound, which can mask stimulusspecific IEG induction. Our experimental paradigm was expressly designed to address this issue. By recording in the dark, we gained the ability to normalize the results by V1 levels, which presumably reflect general arousal effects not specific to the visual modality, probably caused by increased catecholaminergic/cholinergic inputs (Hasselmo, 1995). Alternatively, the data may suggest that $\mathrm{V} 1$ receives spatio-tactile information from indirect connections with S1 and HP (Lavenex and Amaral, 2000), reflecting cross-modal interactions known to occur in the primary sensory cortices (Cohen et al., 1997; Zhou and Fuster, 2000).

To the extent that persistent changes in firing rates indicate mnemonic processing (Fuster and Jervey, 1981), and that arc and zif-268 transcriptional upregulation correlates with synaptic remodeling (Guzowski et al., 2000; Jones et al., 2001), our data support the notion that memories produced by novel stimulation during WK are reactivated during SWS and stored during REM sleep, in a two-step consolidation process (Ribeiro and Nicolelis, 2004) otherwise similar to one-step memory reconsolidation (Nader, 2003; Stickgold and Walker, 2005). This general model does not refer only to hippocampo-cortical processing, but rather to molecular and cellular mechanisms that likely apply to both procedural and declarative memories (Ribeiro and Nicolelis, 2004). This model, which emphasizes the functional complementarity of the different sleep states (Giuditta, 1985; Pavlides and Ribeiro, 2003), is compatible with the evidence that SWS and REM sleep have synergistic effects on human procedural learning (Mednick et al., 2003), and with the downregulation during REM sleep of NGFI-B (Ribeiro et al., 1999), a transcription factor induced by glucorticoids during consolidation but not reconsolidation (von Hertzen and Giese, 2005).

The lack of correlation between time spent in REM sleep and IEG expression indicates that REM sleep plays a brief and mostly permissive role on IEG upregulation, in line with the fact that birds and crocodilians display hundreds of seconds long REM sleep episodes overnight (Siegel, 1995). The notion of the REM sleep episodes as snapshots of IEG upregulation, with SWS functioning as a stop signal, may explain some contradictory findings regarding the weak correlations between learning and total time spent in REM sleep for spatial/episodic memories or comparisons across species (Gais and Born, 2004; Plihal and Born, 1999; Siegel, 1995, 2001). The minutes-long duration of mammalian REM sleep episodes (Siegel, 1995) may have co-evolved with a secondary function of REM sleep in mammals, in addition to IEG upregulation (Ribeiro and Nicolelis, 2004). The reduced dispersion of the number of REM sleep episodes in the last hour across REM (+) animals, as well as the small sample size in this group $(n=4)$, precluded a reliable test of the hypothesis that IEG levels are proportional to the number of times animals enter REM sleep, rather than to the total amount of time spent in REM sleep. Further investigation of the idea that REM sleep functions as a discrete switch for IEG upregulation is in order.

Experience-dependent IEG reinduction during REM sleep is at variance with studies showing that sleep downregulates the expression of IEGs such as zif-268 and c-fos (Basheer et al., 1997; Cirelli and Tononi, 1998, 2000a; O'Hara et al., 1993; Pompeiano et al., 1992, 1994). The discrepancy is partially explained by the fact that these studies did not attempt to sort the separate contributions of SWS and REM sleep to IEG expression. Our previous (Ribeiro et al., 1999, 2002) and current results (Figures 4A and 4B) confirm that SWS induces a marked decrease in IEG expression. Furthermore, the studies mentioned above have only examined negative controls, i.e., animals not exposed to novel experience prior to sleep. In these animals IEG expression remains low, as shown in Figure $\mathrm{S} 1$ of Supplemental Data and previous studies of our own (Ribeiro et al., 1999, 2002). In contrast, IEG levels are strongly reinduced in animals first exposed to novelty/learning and then allowed to enter REM sleep (Ribeiro et al., 1999, 2002; Ulloor and Datta, 2005). An important finding of the present study is the fact that IEG upregulation during REM sleep is stronger in the cortical circuits most activated by previous experience, i.e., IEG reinduction after novel tactile stimulation in the dark is much stronger in $\mathrm{S} 1$ than in V1. This means that our data are compatible with synaptic downscaling during SWS (Tononi and Cirelli, 2003), as long as downscaling occurs preferentially in non-activated circuits. The combination of synaptic upscaling in activated circuits and synaptic downscaling in non-activated circuits is probably what occurs in reality, providing an "èmbossing" mechanism for the selective processing of relevant memories during sleep, and increasing the signal-to-noise ratio of mnemonic consolidation.

In principle, upregulation of IEG transcription during REM sleep should promote neural plasticity in both directions within the neuron, respectively effecting retrograde and anterograde changes capable of stabilizing and propagating memories in a milieu free of interference. IEG upregulation after a few (Ribeiro et al., 1999, 2002) or many (Figure 4) sleep cycles is a plausible explanation for late waves of arc and zif-268 gene expression upregulation many hours after memory acquisition (Bekinschtein et al., 2007; Grimm and Tischmeyer, 1997). The involvement of both IEGs with the regulation of calcium-calmodulin kinase II (Donai et al., 2003; Takeuchi et al., 2002) may underlie the cortical requirement of this kinase for longterm memory (Frankland et al., 2001).

The importance of IS for learning and memory is supported by data showing that rats trained in a conditioned avoidance task have $180 \%$ more IS than controls during the initial post-exposure hours (Datta, 2000). Similar results were also observed after exposure to novel objects (Schiffelholz and Aldenhoff, 2002). In the case of the first study (Datta, 2000), a significant correlation was found between learning and the density of pontine waves, which are abundant both during IS and REM sleep. The same research group, using the two-way active-avoidance learning paradigm, showed that the increase in pontine-wave density during post-training REM sleep is positively correlated with the increased levels of phosphorylated CAMP response element-binding protein (CREB), brain-derived nerve growth factor (BDNF), and Arc protein in the dorsal hippocampus (Ulloor and Datta, 2005). Therefore, it is possible that pontine waves play a key role in the upregulation of IEG expression during sleep, as previously suggested (Ribeiro and Nicolelis, 2004; Ulloor and Datta, 2005) 
The significant correlation between arc cortical expression and spindle-range LFP oscillations (Figure 4D) indeed suggests that the spindle-rich state IS may be causally related to IEG expression, but SWS that immediately precedes IS also shows a great abundance of spindles (Gervasoni et al., 2004; Glin et al., 1991). Spindle-range LFP activity in itself is certainly not sufficient to induce IEG expression, or else SWS and IS would also lead to IEG upregulation, like REM sleep does. On the other hand, rats in the REM $(+)$ group were the only ones that were allowed uninterrupted sleep in the last 60 minutes of the experiment, in contrast with the IS (+) and SWS (+) groups, in which sleep was repeatedly interrupted to prevent animals to enter specific states. Thus, the upregulation of IEG expression detected in the REM (+) group may reflect "consolidated sleep" effects, rather than mechanisms restricted to REM sleep. The most parsimonious conclusion at present is that a full sleep cycle is necessary for IEG reinduction following novel experience.

The putative mechanisms by which sleep spindles may contribute to IEG upregulation remain unknown. Spindles are forebrain neural oscillations (Contreras et al., 1997) associated with behavioral learning (Gais et al., 2002) and long-term potentiation (Rosanova and Ulrich, 2005). The correlation between cortical arc expression and LFP oscillations in the spindle-range (Figure 4D) is compatible with the notion that memory transfer along the hippocampal-cortical axis occurs over long periods of time during post-experience SWS, by way of the synchronization of large-amplitude oscillations such as cortical spindles and hippocampal ripples (Buzsaki, 1996; Sirota et al., 2003).

Our model complements this view by postulating that the persistence of plasticity for hours in the cortex but only minutes in the hippocampus may be an effective mechanism for hippocampo-cortical memory transfer. We also provide empirical support for the synaptic reentry reinforcement (SRR) theory, which postulates that post-learning sleep upregulates activity-dependent and calcium-dependent mechanisms underlying learning (Wittenberg and Tsien, 2002; Wittenberg et al., 2002). But the SRR theory predicts that hippocampo-cortical interactions last for weeks, while our previous (Ribeiro et al., 1999, 2002) and current data indicate that the sleep-dependent relationship between neuronal activity and synaptic remodeling persists for many hours only in the cortex. Indeed, hippocampal firing rate reactivation following novel experience (Pavlides and Winson, 1989, present data) seems to fade quickly over time so as to reach non-significant levels after a few sleep episodes. Likewise, the first REM sleep episode after novel experience induces IEG upregulation in both the hippocampus and the cortex (Ribeiro et al., 1999), but the $\sim 28$ th post-experience episode triggers IEG reinduction in the cortex only (present data). Our results suggest that IEG expression is more strongly coupled to LFPs in S1 than in HP. The fast hippocampal disengagement from sleep-induced plasticity may be due to a specific hippocampal sensitivity to neurochemicals abundant during REM sleep, such as acetylcholine (Hasselmo, 1999) or cortisol (Born and Wagner, 2004; Payne and Nadel, 2004). Alternatively, the very fact that the hippocampal network is more removed from sensory inputs than the primary sensory cortices may cause weaker memory encoding in the hippocampus than in the cortex. Furthermore, the short lifespan of significant changes in hippocampal firing rates and IEG expression levels does not necessarily mean that the transfer of information to the cerebral cortex has been completed after this period. It has been argued that such transfer requires a continued low level of interleaved replay over long periods of time, in which case one would not expect to detect major hippocampal changes through most of the hippocampocortical transfer process (McClelland et al., 1995). Indeed, subtler rate-normalized patterns of neuronal ensemble activity have been shown to persist in the hippocampus for up to 48 hours (Ribeiro et al., 2004).

Whichever the mechanisms underlying early hippocampal disengagement from post-novelty plasticity, the available evidence indicates that novel stimulation is followed by a few sleep-induced plasticity waves in the hippocampus (Ribeiro et al., 1999, 2002), but many in the cortex (present results) (Figure 6A). Nearly nothing is known about the effects of cumulative waves of neural plasticity on memory consolidation. In particular, it is

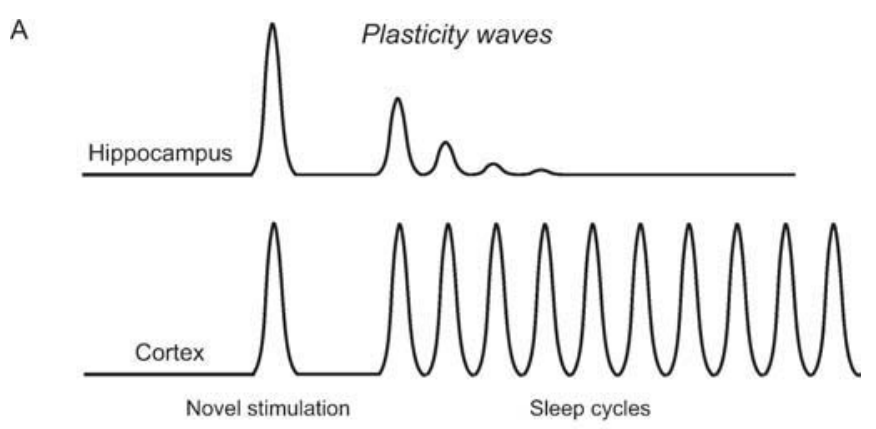

B

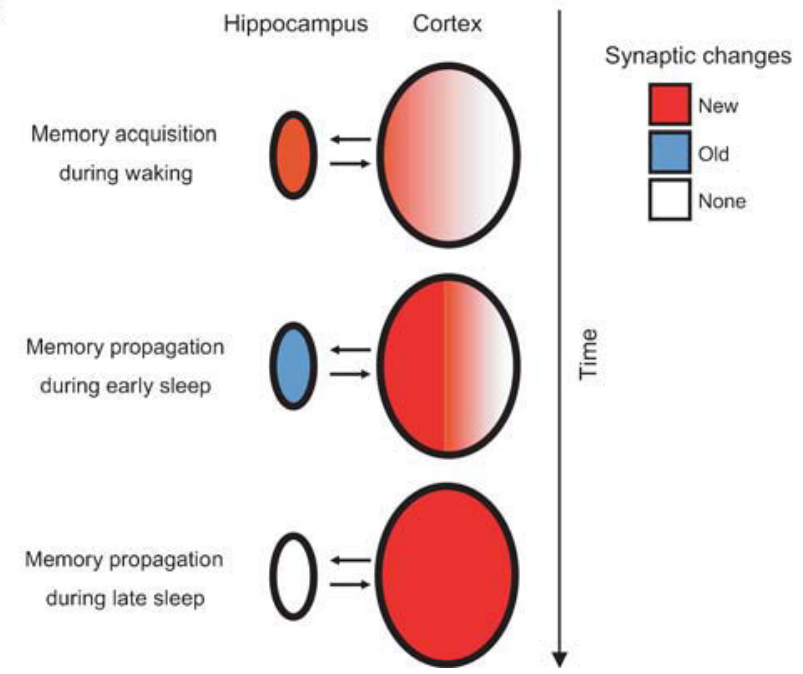

Figure 6. Modeling the hippocampo-cortical dynamics of memory processing. (A) Current and previous results (Ribeiro et al., 1999, 2002) indicate that the hippocampus undergoes a few plasticity waves before fading out. These plasticity waves are probably enough for memories to remain in the hippocampus for weeks or months (Bontempi et al., 1999). In contrast, the cerebral cortex undergoes plasticity waves for a much longer period of time, leading to many more cycles of memory reinforcement and years-old memories (Frankland and Bontempi, 2005). (B) Model of memory propagation from hippocampus to cortex during sleep. By the way of thalamo-cortical inputs (not shown), episodic and spatial memories are acquired during waking as new synaptic changes (red) distributed over hippocampo-cortical networks of neurons (top panel). The recurrence of cortical plasticity during subsequent sleep causes the stabilization and propagation of new synaptic changes in the cortex. Conversely, the fast decay of sleep-dependent plasticity in the hippocampus generates a net outflow of information, gradually flushing memories to associated cortical networks over time (middle and bottom panels).

unclear whether consecutive plasticity waves would result in more durable effects, or simply more intense ones. Still, it seems reasonable to suppose that if a single plasticity wave has effects that last for days, plasticity disengagement of the hippocampus in the range of minutes may result in a corresponding physiological disengagement after weeks or months (Bontempi et al., 1999; Frankland and Bontempi, 2005). Our data add to the notion that synaptic changes initially stored in the hippocampo-cortical loop migrate toward associated cortical networks as sleep recurs (Figure 6B) (Pavlides and Ribeiro, 2003; Ribeiro and Nicolelis, 2004; Ribeiro et al., 2002). According to this model, the sleep-dependent anterograde propagation of synaptic changes would strengthen cortico-cortical connections and cause memory traces to gradually reach farther away from the original neuronal circuits initially involved in memory encoding. This maturation process, characterized by cyclic consolidation and propagation, may be at the roots of the slow learning produced by the object familiarization task, in which a significant reduction in the amount of time exploring the 


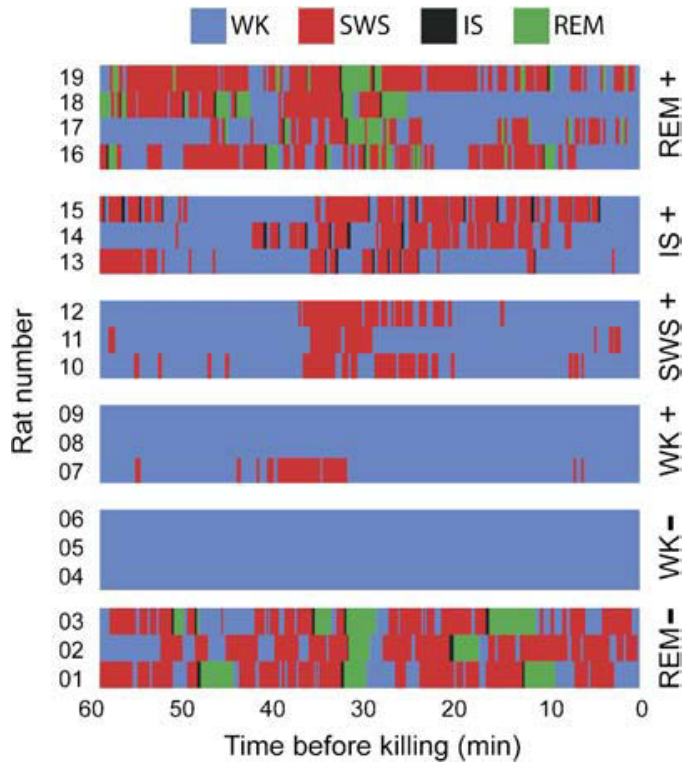

Figure 7. Hypnograms. Shown is the sleep-wake architecture of the last hour of the experiment in the 19 animals in which IEG expression was assessed. Group assignments are shown on the right. Note that state contamination was prevented in all animals but rat 7, which displayed some SWS during the periods that preceded and followed novel stimulation (in the WK+ group, exposure to novel objects occurred between 30 and 10 minutes before killing).

same objects only occurs after several daily sessions (Figure 1B). As a result, memories would be modified over and over again in a dynamic manner, becoming progressively more ingrained at every sleep cycle. We postulate a sleep-dependent process capable of propagating memories both between and inside brain structures, i.e., a process that would extend memories from primary areas to higher-order associative areas, while at the same time increasing the branchness of engrams within each brain region. In principle, such a process should render memories less susceptible to decay, more resistant to interference, and more complexly interconnected with other circuits.

The molecular and cellular model of sleep-dependent memory maturation proposed here accounts for deeper cortical encoding as memories age (Cermak and Craik, 1979; Craik and Lockhart, 1972; Hebb, 1942; McClelland et al., 1995; Marr, 1970; Paller, 1997), and for incremental learning over multiple nights after memory trace acquisition (Stickgold et al., 2000; Walker et al., 2003). By the same token, the fast decay of hippocampal plasticity after novel experience potentially explains the progressive disengagement of the hippocampus in the encoding of remote memories (Bontempi et al., 1999; Corkin et al., 1997; Frankland et al., 2001; Haist et al., 2001; Kim et al., 1995; Lavenex and Amaral, 2000; McGaugh, 2000; Mishkin, 1978; Penfield and Milner, 1958; Quillfeldt et al., 1996; Scoville and Milner, 1957; Winocur et al., 2001; Zola-Morgan and Squire, 1993; Zola-Morgan et al., 1982).

\section{CONFLICT OF INTEREST STATEMENT}

The authors declare that the research was conducted is the absence of any commercial or financial relationships that should be construed as a potential conflict of interest.

\section{ACKNOWLEDGEMENTS}

Work supported by a Pew Latin American Fellowship to SR, an INSERM Fellowship to DG, and NIH grants to MALN. We thank G. Lehew and J. Meloy for electrode manufacturing; E. Jarvis and all lab members for generous access to his laboratory; C. Mello and T. Velho for reagents; I. Araujo for valuable discussions; I. Araujo and J. Ross for help with data analysis, J. Pantoja for help with histology and figure preparation, and L. Oliveira, S. Halkiotis, T. Jones and G. Wood for lab support. Dedicated to Alberto Santos Dumont.

\section{REFERENCES}

Basheer, R., Sherin, J. E., Saper, C. B., Morgan, J. I., and McCarley, R. W. (1997). Effects of sleep on wake-induced c-fos expression. J. Neurosci. 17, 9746-9750.

Bekinschtein, P., Cammarota, M., Igaz, L. M., Bevilaqua, L. R., and Izquierdo I. (2007). Persistence of long-term memory storage requires a late protein synthesis- and BDNF- dependent phase in the hippocampus. Neuron 53, 261-277.

Bontempi, B., Laurent-Demir, C., Destrade, C., and Jaffard, R. (1999). Timedependent reorganization of brain circuitry underlying long-term memory storage. Nature 400 , 671-675.

Born, J., and Wagner, U. (2004). Memory consolidation during sleep: role of cortisol feedback. Ann. NY Acad. Sci. 1032, 198-201.

Bozon, B., Kelly, A., Josselyn, S. A., Silva, A. J., and Davis, S. (2003). MAPK, CREB and zif268 are all required for the consolidation of recognition memory. Philos. Trans. $R$. Soc. Lond. B Biol. Sci. 358, 805-814.

Buzsaki, G. (1996). The hippocampo-neocortical dialogue. Cereb. Cortex 6, 81-92.

Cermak, L., and Craik, F. (1979). Levels of Processing in Human Memory. (Indianapolis, John Wiley \& Sons).

Cirelli, C., and Tononi, G. (1998). Differences in gene expression between sleep and waking as revealed by mRNA differential display. Brain Res. Mol. Brain Res. 56, 293-305.

Cirelli, C., and Tononi, G. (2000). Differential expression of plasticity-related genes in waking and sleep and their regulation by the noradrenergic system. J. Neurosci. 20 , 9187-9194

Cirelli, C., and Tononi, G. (2000). Gene expression in the brain across the sleep-waking cycle. Brain Res. 885, 303-321.

Clayton, D. F., Huecas, M. E., Sinclair-Thompson, E. Y., Nastiuk, K. L., and Nottebohm, F. (1988). Probes for rare mRNAs reveal distributed cell subsets in canary brain. Neuron 1, 249-261.

Cohen, L. G., Celnik, P., Pascual-Leone, A., Corwell, B., and Falz, L. (1997). Functional relevance of cross-modal plasticity in blind humans. Nature 389, 180-183.

Contreras, D., Destexhe, A., Sejnowski, T. J., and Steriade, M. (1997). Spatiotemporal patterns of spindle oscillations in cortex and thalamus. J. Neurosci. 17, 1179-1196.

Corkin, S., Amaral, D. G., Gonzalez, R. G., Johnson, K. A., and Hyman, B. T. (1997). H.M.'s medial temporal lobe lesion: findings from magnetic resonance imaging. $J$. Neurosci. 17, 3964-3979.

Craik, F., and Lockhart, R. (1972). Levels of processing: A framework for memory research. J. Verb Learn. Verb Behav. 11, 671-684.

Datta, S. (2000). Avoidance task training potentiates phasic pontine-wave density in the rat: A mechanism for sleep-dependent plasticity. J. Neurosci. 20, 8607-8613.

Donai, H., Sugiura, H., Ara, D., Yoshimura, Y., and Yamagata, K. (2003). Interaction of Arc with CaM kinase II and stimulation of neurite extension by Arc in neuroblastoma cells expressing CaM kinase II. Neurosci. Res. 47, 399-408.

Forwood, S. E., Winters, B. D., and Bussey, T. J. (2005). Hippocampal lesions that abolish spatial maze performance spare object recognition memory at delays of up to 48 hours. Hippocampus 15, 347-355.

Frankland, P. W., and Bontempi, B. (2005). The organization of recent and remote memory. Nat. Rev. Neurosci. 6, 119-130.

Frankland, P. W., O'Brien, C., Ohno, M., Kirkwood, A., and Silva, A. J. (2001). AlphaCaMKII-dependent plasticity in the cortex is required for permanent memory. Nature $411,309-313$

Fuster, J. M., and Jervey, J. P. (1981). Inferotemporal neurons distinguish and retain behaviorally relevant features of visual stimuli. Science 212, 952-955.

Gais, S., and Born, J. (2004). Declarative memory consolidation: mechanisms acting during human sleep. Learn. Mem. 11, 679-685

Gais, S., Molle, M., Helms, K., and Born, J. (2002). Learning-dependent increases in sleep spindle density. J. Neurosci. 22, 6830-6834.

Gervasoni, D., Lin, S. C., Ribeiro, S., Soares, E. S., Pantoja, P., and Nicolelis, M. (2004). Global forebrain dynamics predict rat behavioral states and their transitions. J. Neurosci. 24, 11137-11147.

Gilbert, P. E., and Kesner, R. P. (2004). Memory for objects and their locations: the role of the hippocampus in retention of object-place associations. Neurobiol. Learn. Mem. $81,39-45$.

Giuditta, A., ed. (1985). A Sequential Hypothesis for the Function of Sleep. (Stuttgart, Fisher-Verlag). pp. 222-224.

Glin, L., Arnaud, C., Berracochea, D., Galey, D., and Jaffard, R. (1991). The intermediate stage of sleep in mice. Physiol. Behav. 50, 951-953.

Gottesmann, C. (1996). The transition from slow-wave sleep to paradoxical sleep: evolving facts and concepts of the neurophysiological processes underlying the intermediate stage of sleep. Neurosci. Biobehav. Rev. 20, 367-387.

Grimm, R., and Tischmeyer, W. (1997). Complex patterns of immediate early gene induction in rat brain following brightness discrimination training and pseudotraining Behav. Brain Res. 84, 109-116.

Gutwein, B. M., Shiromani, P. J., and Fishbein, W. (1980). Paradoxical sleep and memory: long-term disruptive effects of Anisomycin. Pharmacol. Biochem. Behav. 12 377-384. 
Guzowski, J. F., Lyford, G. L., Stevenson, G. D., Houston, F. P., and McGaugh, J. L. (2000). Inhibition of activity-dependent arc protein expression in the rat hippocampus impairs the maintenance of long-term potentiation and the consolidation of long-term memory. J. Neurosci. 20, 3993-4001.

Guzowski, J. F., McNaughton, B. L., Barnes, C. A., and Worley, P. F. (1999). Environmentspecific expression of the immediate-early gene $A r c$ in hippocampal neuronal ensembles. Nat. Neurosci. 2, 1120-1124.

Guzowski, J. F., Setlow, B., Wagner, E. K., and McGaugh, J. L. (2001). Experiencedependent gene expression in the rat hippocampus after spatial learning: a comparison of the immediate-early genes Arc, c-fos, and zif268. J. Neurosci. 21, 5089 5098.

Haist, F., Bowden Gore, J., and Mao, H. (2001). Consolidation of human memory over decades revealed by functional magnetic resonance imaging. Nat. Neurosci. 4 $1139-1145$.

Hasselmo, M. E. (1995). Neuromodulation and cortical function: modeling the physiological basis of behavior. Behav. Brain Res. 67, 1-27.

Hasselmo, M. E. (1999). Neuromodulation: acetylcholine and memory consolidation. Trends Cogn. Sci. 3, 351-359.

Hebb, D. 0. (1942). The effects of early and late brain injury upon test scores, and the nature of normal adult intelligence. Proc. Am. Philos. Soc. 85, 275-292.

Hebb, D. O. (1949). The Organization of Behavior: A Neuropsychological Theory. (New York, John Wiley \& Sons)

Hirase, H., Leinekugel, X., Czurko, A., Csicsvari, J., and Buzsaki, G. (2001). Firing rates of hippocampal neurons are preserved during subsequent sleep episodes and modified by novel awake experience. Proc. Natl. Acad. Sci. USA 98, 9386-9390.

Izquierdo, I., and Medina, J. H. (1997). Memory formation: the sequence of biochemica events in the hippocampus and its connection to activity in other brain structures. Neurobiol. Learn. Mem. 68, 285-316.

Ji, D., and Wilson, M. A. (2007). Coordinated memory replay in the visual cortex and hippocampus during sleep. Nat. Neurosci. 10, 100-107.

Jones, M. W., Errington, M. L., French, P. J., Fine, A., and Bliss, T. V. (2001). A requirement for the immediate early gene Zif268 in the expression of late LTP and long-term memories. Nat. Neurosci. 4, 289-296.

Kim, J. J., Clark, R. E., and Thompson, R. F. (1995). Hippocampectomy impairs the memory of recently, but not remotely, acquired trace eyeblink conditioned responses. Behav. Neurosci. 109, 195-203.

Kralik, J., Dimitrov D, Krupa, D., Katz, D., and Cohen, D. (2001). Techniques for long-term multisite neuronal ensemble recordings in behaving animals. Methods 25, 121-150.

Kudrimoti, H. S., Barnes, C. A., and McNaughton, B. L. (1999). Reactivation of hippocampal cell assemblies: effects of behavioral state, experience, and EEG dynamics. J. Neurosci. 19, 4090-4101.

Lavenex, P., and Amaral, D. G. (2000). Hippocampal-neocortical interaction: a hierarchy of associativity. Hippocampus 10, 420-430.

Logothetis, N. K., Pauls, J., Augath, M., Trinath, T., and Oeltermann, A. (2001). Neurophysiological investigation of the basis of the fMRI signal. Nature 412, 150-157.

Lopes da Silva, F. (1991). Neural mechanisms underlying brain waves: from neural membranes to networks. Electroencephalogr. Clin. Neurophysiol. 79, 81-93.

Louie, K., and Wilson, M. A. (2001). Temporally structured replay of awake hippocampal ensemble activity during rapid eye movement sleep. Neuron 29, 145-156.

Lyford, G. L., Yamagata, K., Kaufmann, W. E., Barnes, C. A., and Sanders, L. K. (1995). $A r c$, a growth factor and activity-regulated gene, encodes a novel cytoskeletonassociated protein that is enriched in neuronal dendrites. Neuron 145, 433-434.

Marr, D. (1970). A theory for cerebral neocortex. Proc. R. Soc. Lond. B Biol. Sci. 176, $161-234$

McClelland, J. L., McNaughton, B. L., and Oreilly, R. C. (1995). Why there are complementary learning systems in the hippocampus and neocortex: insights from the successes and failures of connectionist models of learning and memory. Psychol. Rev. 102, 419-457.

McGaugh, J. L. (2000). Memory-a century of consolidation. Science 287, 248-251.

Mednick, S. C., Nakayama, K., and Stickgold, R. (2003). Sleep-dependent learning: a nap is as good as a night. Nat. Neurosci. 6, 697-698.

Mello, C. V., and Clayton, D. F. (1994). Song-induced ZENK gene expression in auditory pathways of songbird brain and its relation to the song control system. J. Neurosci. $14,6652-6666$

Mishkin, M. (1978). Memory in monkeys severely impaired by combined but not by separate removal of amygdala and hippocampus. Nature 273, 297-298.

Mumby, D. G., Tremblay, A., Lecluse, V., and Lehmann, H. (2005). Hippocampal damage and anterograde object-recognition in rats after long retention intervals. Hippocampus 15, 1050-1056.

Nader, K. (2003). Memory traces unbound. Trends Neurosci. 26, 65-72.

Nicolelis, M. A., Dimitrov, D., Carmena, J. M., Crist, R., and Lehew, G. (2003). Chronic, multisite, multielectrode recordings in macaque monkeys. Proc. Natl. Acad. Sci. USA 100, 11041-11046.

Niessing, J., Ebisch, B., Schmidt, K. E., Niessing, M., and Singer, W. (2005). Hemodynamic signals correlate tightly with synchronized gamma oscillations. Science 309 948-951.

Nunez, P. L. (1981). Electric Fields of the Brain: The Neurophysics of EEG. (New York Oxford University Press).

O'Hara, B. F., Young, K. A., Watson, F. L., Heller, H. C., and Kilduff, T. S. (1993). Immediate early gene expression in brain during sleep deprivation: preliminary observations. Sleep 16, 1-7.

0'Keefe, J. (1979). A review of the hippocampal place cells. Prog. Neurobiol. 13, 419-439.

Paller, K. A. (1997). Consolidating dispersed neocortical memories: the missing link in amnesia. Memory 5, 73-88.
Pavlides, C., and Ribeiro, S. (2003). Recent evidence of memory processing in sleep. In: Sleep and Brain Plasticity. Maquet, P., Smith, C., Stickgold, R., ed. (Oxford, UK, Oxford University Press). pp. 327-362.

Pavlides, C., and Winson, J. (1989). Influences of hippocampal place cell firing in the awake state on the activity of these cells during subsequent sleep episodes. J. Neurosci. 9, 2907-2918.

Paxinos, G., and Watson, C. (1997). The Rat Brain in Stereotaxic Coordinates. (San Diego, Academic Press)

Payne, J. D., and Nadel, L. (2004). Sleep, dreams, and memory consolidation: the role of the stress hormone cortisol. Learn. Mem. 11, 671-678.

Peigneux, P., Laureys, S., Fuchs, S., Collette, F., Perrin, F., Reggers, J., Phillips, C. Degueldre, C., Del Fiore, G., Aerts, J., Luxen, A., and Maquet, P. (2004). Are spatia memories strengthened in the human hippocampus during slow wave sleep? Neuron 44, 535-545.

Penfield, W., and Milner, B. (1958). Memory deficit produced by bilateral lesions in the hippocampal zone. Arch. Neurol. Psych. 79, 475-497.

Petersohn, D., Schoch, S., Brinkmann, D. R., and Thiel, G. (1995). The human synapsin II gene promoter. Possible role for the transcription factor zif268/egr-1, polyoma enhancer activator 3, and AP2. J. Biol. Chem. 270, 24361-24369.

Plihal, W., and Born, J. (1999). Effects of early and late nocturnal sleep on priming and spatial memory. Psychophysiology 36, 571-582.

Pompeiano, M., Cirelli, C., and Tononi, G. (1992). Effects of sleep deprivation on fos-like immunoreactivity in the rat brain. Arch. Ital. Biol. 130, 325-335.

Pompeiano, M., Cirelli, C., and Tononi, G. (1994). Immediate-early genes in spontaneous wakefulness and sleep: expression of c-fos and NGIF-A mRNA protein. J. Sleep Res 3, 80-96.

Qin, Y. L., McNaughton, B. L., Skaggs, W. E., and Barnes, C. A. (1997). Memory reprocessing in corticocortical and hippocampo-cortical neuronal ensembles. Philos. Trans. $R$ Soc. Lond. B Biol. Sci. 352, 1525-1533.

Quillfeldt, J. A., Zanatta, M. S., Schmitz, P. K., Quevedo, J., and Schaeffer, E. (1996). Different brain areas are involved in memory expression at different times from training. Neurobiol. Learn. Mem. 66, 97-101.

Ramirez-Amaya, V., Vazdarjanova, A., Mikhael, D., Rosi, S., and Worley, P. F. (2005). Spatial exploration-induced Arc mRNA and protein expression: evidence for selective network-specific reactivation. J. Neurosci. 25, 1761-1768.

Ribeiro, S., Gervasoni, D., Soares, E. S., Zhou, Y., and Lin, S. C., Pantoja, P., Lavine, M. and Nicolelis, M.(2004). Long lasting novelty-induced neuronal reverberation during slow-wave sleep in multiple forebrain areas. PLOS Biol. 2, 126-137.

Ribeiro, S., Goyal, V., Mello, C. V., and Pavlides, C. (1999). Brain gene expression during REM sleep depends on prior waking experience. Learn. Mem. 6, 500-508.

Ribeiro, S., Gervasoni, D., Soares, E. S., Zhou, Y., Lin, S. C., Pantoja, P., Lavine, M., Nicolelis, M. (2002). Induction of hippocampal long-term potentiation during waking leads to increased extrahippocampal zif-268 expression during ensuing rapid-eyemovement sleep. J. Neurosci. 22, 10914-10923.

Ribeiro, S., Nicolelis, M. A. L. (2004). Reverberation, storage and postsynaptic propagation of memories during sleep. Learn. Mem. 11, 686-696.

Rosanova, M., and Ulrich, D. (2005). Pattern-Specific Associative Long-Term Potentiation Induced by a Sleep Spindle-Related Spike Train. Neurosci. 25, 9398-9405.

Schiffelholz, T., and Aldenhoff, J. B. (2002). Novel object presentation affects sleep-wake behavior in rats. Neurosci. Lett. 328, 41-44.

Scoville, W. B., and Milner, B. (1957). Loss of recent memory after bilateral hippocampal lesions. J. Neurol. Neurosurg. Psych. 20, 11-21.

Segawa, M. (2006). Epochs of development of the sleep-wake cycle reflect the modulation of the higher cortical function particular for each epoch. Sleep Biol. Rhythms 4, 16-26.

Sejnowski, T. J., and Destexhe, A. (2000). Why do we sleep? Brain Res. 886, 208223.

Siapas, A. G., and Wilson, M. A. (1998). Coordinated interactions between hippocampal ripples and cortical spindles during slow-wave sleep. Neuron 21, 1123-1128.

Siegel, J. M. (1995). Phylogeny and the function of REM sleep. Behav. Brain. Res. 69, 29-34.

Siegel, J. M. (2001). The REM sleep-memory consolidation hypothesis. Science 294, 1058-1063

Simons, D. J., and Woolsey, T. A. (1979). Functional organization in mouse barrel cortex. Brain Res. 165, 327-332.

Sirota, A., Csicsvari, J., Buhl, D., and Buzsaki, G. (2003). Communication between neocortex and hippocampus during sleep in rodents. Proc. Natl. Acad. Sci. USA 100 2065-2069.

Squire, L. R., Knowlton, B., and Musen, G. (1993). The structure and organization of memory. Annu. Rev. Psychol. 44, 453-495.

Steward, 0., Wallace, C. S., Lyford, G. L., and Worley, P. F. (1998). Synaptic activation causes the mRNA for the IEG Arc to localize selectively near activated postsynaptic sites on dendrites. Neuron 21, 741-751.

Stickgold, R. (1998). Sleep: off-line memory reprocessing. Trends Cogn. Sci. 2, 484492.

Stickgold, R., Hobson, J. A., Fosse, R., and Fosse, M. (2001). Sleep, learning, and dreams: off-line memory reprocessing. Science 294, 1052-1057.

Stickgold, R., and Walker, M. P. (2005). Memory consolidation and reconsolidation: what is the role of sleep? Trends Neurosci. 28, 408-415.

Stickgold, R., Whidbee, D., Schirmer, B., Patel, V., and Hobson, J. A. (2000). Visual discrimination task improvement: a multi-step process occurring during sleep. J. Cogn. Neurosci. 12, 246-254.

Swadlow, H. A. (2003). Fast-spike Interneurons and Feedforward Inhibition in Awake Sensory Neocortex. Cereb. Cortex 13, 25-32.

54 
Takashima, A., Petersson, K. M., Rutters, F., Tendolkar, I., and Jensen, 0. (2006). Declarative memory consolidation in humans: a prospective functional magnetic resonance imaging study. Proc. Natl. Acad. Sci. USA 103, 756-761.

Takeuchi, Y., Miyamoto, E., and Fukunaga, K. (2002). Activation of the rat dopamine D2 receptor promoter by mitogen-activated protein kinase and Ca2+/calmodulindependent protein kinase II pathways. J. Neurochem. 83, 784-796.

Thiel, G., Schoch, S., and Petersohn, D. (1994). Regulation of synapsin I gene expression by the zinc finger transcription factor zif268/egr-1. J. Biol. Chem. 269, 15294-15301.

Tononi, G., and Cirelli, C. (2003). Sleep and synaptic homeostasis: a hypothesis. Brain Res. Bull. 62, 143-150.

Tononi, G., and Cirelli, C. (2003b). Sleep and synaptic homeostasis: a hypothesis. Brain Res. Bull. 62, 143-150.

Ulloor, J., and Datta, S. (2005). Spatio-temporal activation of cyclic AMP response element-binding protein, activity-regulated cytoskeletal-associated protein and brain-derived nerve growth factor: a mechanism for pontine-wave generato activation-dependent two-way active-avoidance memory processing in the rat J. Neurochem. 95, 418-428.

von Hertzen, L. S. J., Giese, K. P. (2005). Memory reconsolidation engages only a subset of immediate-early genes induced during consolidation. J. Neurosci. 25, 19351942.

Walker, M. P., Brakefield, T., Seidman, J., Morgan, A., and Hobson, J. A. (2003). Sleep and the time course of motor skill learning. Learn. Mem. 10, 275-284.

Wallace, C. S., Withers, G. S., Weiler, I. J., George, J. M., and Clayton, D. F. (1995). Correspondence between sites of NGFI-A induction and sites of morphological plas- ticity following exposure to environmental complexity. Brain Res. Mol. Brain Res. 32, $211-220$

Wilson, M. A., and McNaughton, B. L. (1994). Reactivation of hippocampal ensemble memories during sleep. Science 265, 676-679.

Winocur, G., McDonald, R. M., and Moscovitch, M. (2001). Anterograde and retrograde amnesia in rats with large hippocampal lesions. Hippocampus 11, 18-26.

Wisden, W., Errington, M. L., Williams, S., Dunnett, S. B., and Waters, C. (1990). Differential expression of immediate early genes in the hippocampus and spinal cord. Neuron 4, 603-614.

Wittenberg, G. M., Sullivan, M. R., and Tsien, J. Z. (2002). Synaptic reentry reinforcement based network model for long-term memory consolidation. Hippocampus 12 , 637-647.

Wittenberg, G. M., and Tsien, J. Z. (2002). An emerging molecular and cellular framework for memory processing by the hippocampus. Trends Neurosci. 25, 501-505.

Zhou, Y. D., and Fuster, J. M. (2000). Visuo-tactile cross-modal associations in cortical somatosensory cells. Proc. Natl. Acad. Sci. USA 97, 9777-9782.

Zola-Morgan, S., and Squire, L. R. (1993). Neuroanatomy of memory. Annu. Rev. Neurosci. $16,547-563$

Zola-Morgan, S., Squire, L. R., and Mishkin, M. (1982). The neuroanatomy of amnesia: amygdala-hippocampus versus temporal stem. Science 218, 13371339.

doi: 10.3389/neuro.01/1.1.003.2007 
